\title{
Uso y Manejo de las Especies Sembradas en las Chagras de Dos Comunidades Murui-Muinane de la Amazonia Colombiana
}

\author{
Sandra Sierra y Lauren Raz
}

\section{Research}

\section{Resumen}

Para identificar los efectos de la transculturación en las prácticas agrícolas tradicionales asociadas a la "chagra" amazónica, se realizó un estudio comparativo entre dos comunidades de la etnia Murui-Muinane del Amazonas Colombiano: San Rafael (lejos de un centro urbano) y Km. 11 (cerca del casco urbano de Leticia) donde se muestrearon 14 y 11 chagras, respectivamente. Se realizaron caminatas etnobotánicas y colecciones documentando diversidad y abundancia de las especies sembradas (excluyendo malezas). Se encontró 129 y 91 especies respectivamente en San Rafael y el Km. 11 y, se establecieron siete categorías de uso. "Alimentos" fue la categoría más importante en las dos comunidades, seguida por "ventas" (Km. 11) y "medicinal" (San Rafael). La aplicación del índice de saliencia y el índice modificado de Byg \& Balslev resalta las diferencias entre las especies consideradas importantes por las dos comunidades. Los efectos de la transculturación sobre la biodiversidad y sus usos son mucho más notorios en $\mathrm{Km}$. 11 que en San Rafael.

\section{Abstract}

To identify the effects of transculturation on traditional swidden-fallow agriculture in the Amazon (basic unit: the chagra), a comparative study was undertaken in two communities of the ethnicity Murui-Muinane in the Colombian Amazon: San Rafael (far from any urban center) and $\mathrm{Km}$. 11 (near the city of Leticia), where 14 and 11 chagras were sampled, respectively. Ethnobotanical walks and collections were carried out to document diversity and abundance of planted species (excluding weeds). In San Rafael and Km. 11, 129 and 91 species were found, respectively, and seven use categories were identified. Food was the most important category in both communities, followed by income (Km. 11) and medicine (San Rafael). Use of the salience and modified Byg \& Balslev indices highlight differences in the species considered important by the two communities. The effects of transculturation on biodiversity and its uses are much greater in $\mathrm{Km}$. 11 than in San Rafael.

\section{Introducción}

En la amazonia colombiana, el sistema tradicional indígena de agricultura de subsistencia es la chagra, un sistema productivo comunal, también conocido como agricultura itinerante o tumba y quema. Es un sistema policultivo con un ciclo productivo de varios años, después del cual la vegetación natural se regenera (aunque este último proceso también puede ser sujeto a diferentes grados de manejo). Aunque las especies vegetales que se cultivan y el uso que se hace de las mismas varían según el contexto cultural y geográfico, para muchos pueblos amazónic-

\section{Correspondence}

Sandra Sierra, Posgrado en Ciencias-Biología. Biodiversidad y Conservación. Instituto de Ciencias Naturales, Facultad de Ciencias, Universidad Nacional de Colombia, Apartado 7495, Bogotá D.C., COLOMBIA. smsierrav@unal.edu.co

Lauren Raz, Instituto de Ciencias Naturales (ICN), Facultad de Ciencias, Universidad Nacional de Colombia, Apartado 7495, Bogotá D.C., COLOMBIA.

Iraz@unal.edu.co

Ethnobotany Research \& Applications 12:473-495 (2014)

Published: 09 October 2014 
os la chagra es la unidad fundamental de los sistemas de producción de especies alimenticias y medicinales (Castro-S. \& Galán-R. 2003, CIFISAM 2005, Galán 2003, Vélez \& Vélez 1999). El uso y manejo de la chagra, más allá de ser un conjunto de prácticas agrícolas, son procesos estrechamente identificados con una forma de vida entre las comunidades indígenas, e influyen en su pensamiento y cosmovisión (CIFISAM 2005).

El sistema tradicional de la chagra es susceptible a diferentes procesos de transculturación. Influencias ajenas pueden afectar a métodos de siembra, abundancia y diversidad de especies sembradas, entre otros factores. La proximidad de la chagra a un centro urbano puede influir en la diversidad de los cultivos, pero el efecto no es siempre igual. Padoch y De Jong (1991) sugieren que cuanto más lejos está un centro urbano, mayor es la diversidad biológica de dichos cultivos. Por otro lado, Lamont et al. (1999) y Wezel y Ohl (2005) comentan que la cercanía de los centros urbanos aumenta la diversidad de especies de cultivos de corto tiempo. Partiendo de la hipótesis que la transculturación se expresa más notoriamente en comunidades cercanas a centros urbanos (Sierra 2007, Tríana-Moreno et al. 2006, Vélez \& Velez 1999), se espera encontrar diferencias significativas en el uso y manejo de las chagras entre comunidades ubicadas cerca de un centro urbano y las de comunidades más alejadas. Para probar esta hipótesis se eligieron dos comunidades, pertenecientes a la misma etnia, pero que habitan dos zonas distintas y alejadas entre sí, en el departamento del Amazonas. La primera comunidad, de San Rafael, se encuentra a considerable distancia de los dos centros poblados más cercanos: El Estrecho, Loreto en Perú y Puerto Leguízamo, Putumayo en Colombia. La segunda comunidad, de Km. 11 de Leticia, está ubicada a tan sólo 11 kilómetros de Leticia, la capital del departamento del Amazonas en Colombia.

En el presente trabajo se pretenden establecer qué diferencias significativas existen en el uso y manejo de las especies sembradas en las chagras entre las dos comunidades indígenas estudiadas. De esta manera, se busca contestar las siguientes preguntas: ¿Se encuentran diferencias en la riqueza de las especies sembradas entre las dos comunidades?, ¿Cuál es el uso que le dan las personas de las dos comunidades a las especies que tienen sembradas en sus chagras?, ¿Cuál es la especie sembrada más importante? ¿Existe una relación entre las especies nombradas como más importantes y las especies que realmente tienen sembradas en sus chagras?

\section{Área de estudio}

Este estudio se llevó a cabo en dos comunidades indígenas de la etnia Murui-Muinane (Uitoto), ubicadas en San Rafael del Caraparaná en el Corregimiento departamental del Encanto, Amazonas (141'4.5”S, 73¹4'22.3”W), y la comunidad Nimaira Naimekeibiri que habita en el
Km. 11 de Leticia en el departamento del Amazonas (407'34.38"S, 6958'17.41"W (Figura 1). La denominación Uitoto (Huitoto, Witoto) se refiere a un grupo étnico cuyo territorio incluye, en el occidente, el río Putumayo y sus afluentes, y se extiende en el oriente hasta el medio Caquetá. La denominación Murui se aplica tradicionaImente a la gente de la zona occidental de este territorio y los del oriente son Muinane (pero los Uitoto-Muinane no pertenecen a la etnia Muinane, quienes hablan otro idioma). La denominación Murai también fue registrada durante el trabajo de campo, y se considera sinónimo de Murui.

La comunidad de San Rafael del Caraparaná cuenta con una población aproximada de 400 habitantes, en su gran mayoría se denominan Murui. Se encuentra ubicada en el sudoeste del departamento del Amazonas, sobre el Río Caraparaná, afluente del Río Putumayo. La comunidad pertenece al Resguardo Predio Putumayo (CORPOAMAZONÍA 2008). Sus ingresos económicos se derivan principalmente del suministro de alimentos y otros productos al internado de la comunidad. San Rafael se encuentra en cercanías a la comunidad de San José (Antiguo centro de la Casa Arana) y a unos días de camino de la comunidad de la Chorrera, centro cultural de los Murui.

La comunidad Nimaira Naimekeibiri- Km. 11 de Leticiacuyo significado es "Patio de la Ciencia Dulce", tiene una población aproximada de 225 habitantes Murui-Muinane y se autodenominan Uitotos en su mayoría (Nieto 2006). Esta comunidad se ubica cerca a la ciudad de Leticia, Amazonas, en el Kilómetro 11 de la carretera LeticiaTarapacá. Pertenece al resguardo Ticuna-Uitoto Km. 6 y 11, que ocupa un área de más o menos 750 hectáreas (CORPOAMAZONÍA 2008, Gasca A. 2005). Fue creada en el año 1962 por hijos de los indígenas desplazados después del genocidio de la cauchería en la Casa Arana.

\section{Métodos}

\section{Fase de campo}

En el periodo comprendido entre septiembre de 2009 y enero de 2010 , se realizaron visitas previas a las comunidades de San Rafael y del Km. 11 de Leticia, permitiendo así un consentimiento informado y planificación de las actividades con los líderes y conocedores locales nombrados por la comunidad.

\section{Estudio florístico}

Durante el trabajo se tuvieron en cuenta principalmente chagras con tamaños de media hectárea en adelante: catorce chagras ubicadas en la comunidad de San Rafael y once en la comunidad Km. 11 de Leticia. En el centro de la chagra de cada uno de los conocedores locales, se hicieron dos levantamientos rectangulares de $40 \times 2 \mathrm{~m}$ 


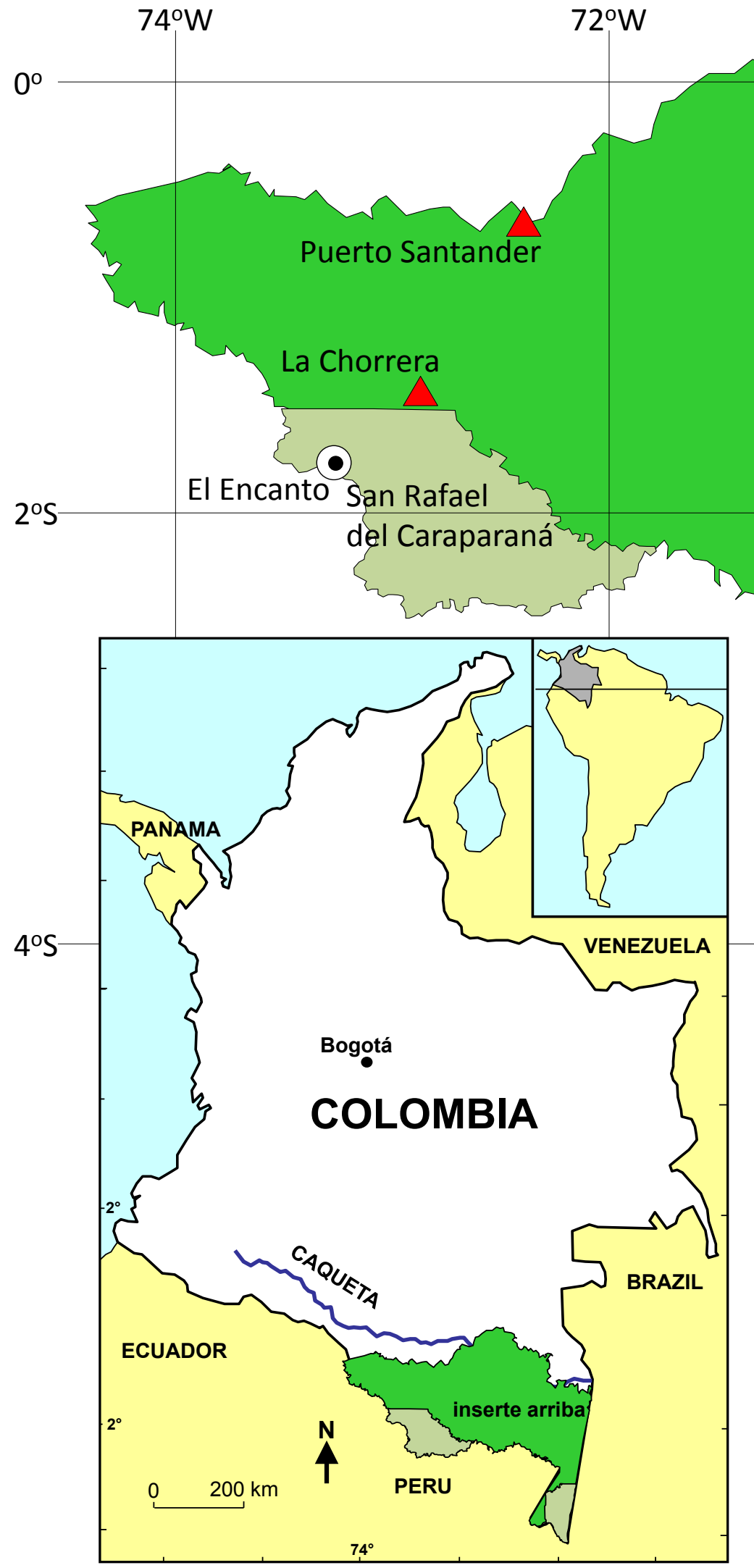

$70^{\circ} \mathrm{W}$

Pacoa

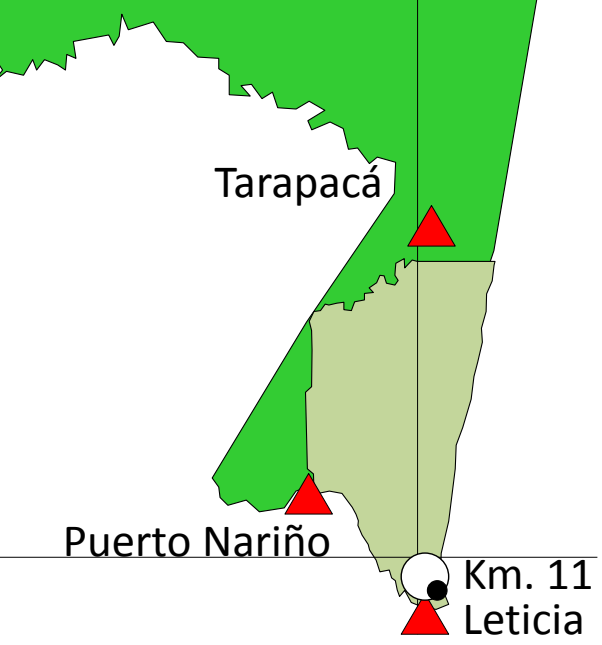

Figura 1. Ubicación de las comunidades Estudiadas (Comunidad San Rafael del Caraparaná, El Encanto, y Comunidad Km. 11. de Leticia), Amazonas, Colombia. 
(80 $\left.\mathrm{m}^{2}\right)$, dispuestos en forma de "L". Con excepción de una chagra de la Comunidad del Km. 11 de Leticia, que por su reducido tamaño (menos de media hectárea) sólo permitió un único levantamiento. En cada uno de los levantamientos se tomaron datos de riqueza y abundancia para todas las especies presentes en el transecto. Se realizaron colecciones botánicas de las especies cultivadas y de las especies silvestres no consideradas malezas. Como complemento del estudio se realizaron caminatas etnobotánicas en cada una de las chagras para registrar el conocimiento tradicional.

La determinación de los ejemplares botánicos se realizó usando claves, descripciones y revisiones taxonómicas (Bernal et al. 2006, Gentry 1993, Ribeiro et al. 1999, Rudas-L. \& Prieto-C. 2005, Vásquez-M. 1997). El material botánico fue determinado y luego depositado en el Herbario Nacional Colombiano (COL), del Instituto de Ciencias Naturales de la Universidad Nacional de Colombia en Bogotá. El material botánico fue etiquetado bajo la numeración de Sandra Milena Sierra Vega (SMS 001 a SMS 572). Para verificar la validez de los nombres científicos se recurrió a la base de datos electrónica Trópicos del Jardín Botánico de Missouri (Tropicos 2014) y se consultó a Brummitt \& Powell (1992) y The Plant List (2013) para citar los nombres de los autores.

\section{Entrevistas}

Para caracterizar el uso de especies encontradas en las chagras, se implementó un plan de trabajo aplicando metodologías de entrevistas semi-estructuradas (Cunningham 2001) y de observación participativa (Bonilla \& Rodríguez 2005). La recopilación de la información (entrevistas y visita a las chagras) se realizó con los miembros de cada grupo familiar que participó en el estudio (Cunningham 2001, Vélez \& Vélez 1999). Las entrevistas semiestructuradas se realizaron a los propietarios de las chagras: en total 13 entrevistas en la comunidad de San Rafael y 11 en el Km. 11 de Leticia. En la comunidad de San Rafael, sólo se realizaron trece entrevistas, ya que una de las chagras es comunal y por lo tanto no tiene responsable específico.

Las entrevistas se realizaron simultáneamente durante los levantamientos y las caminatas etnobotánicas. Durante las entrevistas se registraron los siguientes datos por especie: nombre común, nombre indígena (si tenía), uso, partes de la planta usadas, forma de uso, lugar de siembra, destino final del producto y otros datos aleatorios que surgieron como respuesta a las preguntas que se hicieron en el transcurso de las mismas (Albuquerque et al. 2010, Jiménez Escobar 2012, Jiménez Escobar et al. 2011, Karremans 1994).

\section{Fase de análisis}

Para facilitar la comparación entre las comunidades estudiadas, la importancia relativa de las especies fue medida empleando índices etnobotánicos de valor de uso, saliencia y valor de importancia. Estos valores aunque son cualitativos, se tuvieron en cuenta con datos cuantitativos dentro del estudio.

\section{Valor de uso}

La metodología utilizada para determinar el valor de uso de las especies encontradas en las chagras fue la de Sumatoria de Usos (Boom 1990, Galeano 2000, Paz Y Mino C. et al. 1995, Phillips 1996, Phillips \& Gentry 1993). En esta metodología, el número de usos es sumado dentro de cada categoría de uso, para evaluar el valor de uso de una especie. Esta es la forma más rápida de cuantificar datos etnobotánicos (Boom 1990, Marin et al. 2005). Este enfoque plantea que cada uso mencionado para una especie determinada contribuye al valor total de importancia de dicha especie, independientemente de la categoría. Aunque el número de usos registrados puede ser más un efecto del esfuerzo de investigación que de la importancia relativa de cada especie (o tipo de vegetación), y aunque esta metodología tampoco es un indicador de la importancia cultural de cada uso, sí determina cuales son las especies que tienen mayor diversidad de usos y facilita comparaciones entre dos o más comunidades (Sánchez et al. 2001). Los usos registrados en las entrevistas y durante la colección de muestras en los levantamientos en cada comunidad fueron asignadas posteriormente a categorías de uso con base en las descritas en Marín-Corba et al. (2005), Cárdenas et al. (2002) y complementadas por Gausset (2004) y Jiménez Escobar et al. (2009). Una vez sumados los Valores de Uso, se calculó los intervalos de confianza encontrados para cada comunidad en el programa estadístico $R$ ( $R$ Software 2011). Se realizó un gráfico en forma de caja de Tukey, en el cual se explican los datos ubicados en la caja bajo el percentil del $25 \%$ al $75 \%$. La barra oscura dentro de la imagen, representa la media de los datos (Figura 2).

\section{$\underline{\text { Índice de saliencia cultural }}$}

El Índice de saliencia cultural (Albuquerque et al. 2010, Hoffman \& Galleher 2007) fue calculado empleando el programa Anthropac 4.0 (Borgati \& Natick 1996). Este índice es usado para calcular la frecuencia y orden de respuestas en listas libres (Quinlan et al. 2002), en este caso respondiendo a la pregunta "¿Qué especie sembrada es más importante para usted?" Este índice fue empleado para poder incluir las percepciones de las personas sobre las especies sembradas. Adicionalmente se busca explicar porqué una especie es abundante o no en cada una de las comunidades y así probar la hipótesis que la especie más abundante es la más saliente. 


\section{Sierra \& Raz - Uso y Manejo de las Especies Sembradas en las Chagras de Dos Comunidades Murui-Muinane de la Amazonia Colombiana}

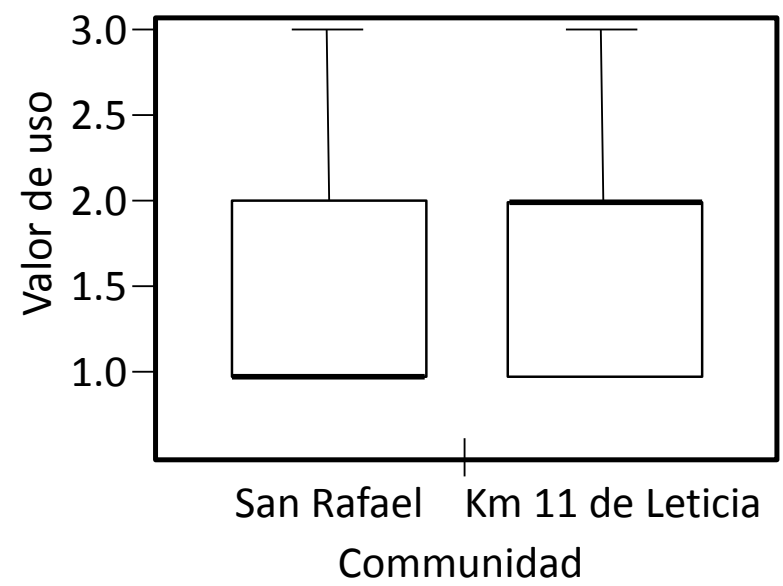

Figura 2. Valor de uso (VU) de las comunidades de San Rafael y de la comunidad Km. 11 de Leticia, Colombia.

Para observar si existe una relación directamente proporcional entre las especies colectadas y las mencionadas como importantes, se realizó una regresión lineal directa, la cual tuvo en cuenta la abundancia de las especies colectadas en cada una de las chagras vs. la saliencia, que se obtuvo por las especies más nombradas en cada una de las dos comunidades. Si una especie tiene el índice de saliencia alto y una abundancia alta, es considerada una especie importante para dicha comunidad, porque fue nombrada como una de las especies más importantes en sus chagras por la gran mayoría de personas entrevistas; por otro lado, si una de las especies tiene una saliencia baja, y sus abundancias son bajas, es considera una especie menos importante, ya que es una de las especies menos nombradas por las personas de sus comunidades. Aunque la regresión lineal explica los datos, hay que tener en cuenta que no hay causa-efecto entre saliencia y abundancia, aunque los datos así se presenten.

\section{Índice modificado valor importancia de Byg \& Balslev}

El índice de valor de importancia de Byg \& Balslev (2001) pretende identificar mediante un consenso cuantitativo, las especies más importantes para una comunidad. Este consenso permite determinar una medida del porcentaje de informantes, que mencionan una especie. Este índice se calcula con la fórmula IVs $=(N i s / n)$, donde Nis = número de informantes de cada comunidad que mencionaron una especie como la más sembrada (frecuencia de mención) y $n=$ número total de informantes por comunidad. En esta implementación se preguntó por la especie más sembrada en vez de la "más importante" ya que el análisis de saliencia mide "importancia" mientras que esta modificación del índice de Byg \& Balslev permite comparar las especies nombradas como las más sembradas con los datos de abundancia recolectados en los transectos.

Con el fin de establecer los rangos para el grado de consenso dentro del índice, se usó la fórmula de Sturges (1926) $K=1+3,3 \log (n)$ que permite estandarizar los datos. Para hacerlo se divide la tabla en niveles (muy alto, alto, medio, bajo y ninguno).

\section{Resultados}

\section{Estudio florístico}

En la comunidad de San Rafael, se realizaron levantamientos florísticos en 14 chagras (incluida la chagra comunal, en la cual no se realizó entrevista, sino sólo los levantamientos florísticos). Como resultado se registraron un total de 129 especies distribuidas en 74 géneros y 60 familias. En la comunidad del Km. 11 de Leticia, se realizaron levantamientos florísticos en 11 chagras y se registraron un total de 91 especies, distribuidas en 60 géneros y 46 familias (Tabla 1).

La familia con la mayor cantidad de géneros en las dos comunidades es la familia Poaceae con cuatro géneros (Cymbopogon, Oryza, Saccharum, Zea), seguida por las familias Solanaceae, en San Rafael, con tres géneros (Solanum, Nicotiana, Capsicum) y la familia Arecaceae, en la comunidad del $\mathrm{Km}$. 11, con cuatros géneros (Bactris, Euterpe, Mauritia, Oenocarpus). El porcentaje de fa-

Tabla 1. Composición florística general de las especies colectadas.

\begin{tabular}{|l|c|c|c|c|c|c|}
\hline \multirow{2}{*}{ Taxones } & \multicolumn{2}{|c|}{ Familias } & \multicolumn{2}{c|}{ Géneros } & \multicolumn{2}{c|}{ Especies } \\
\cline { 2 - 7 } & San Rafael & $\mathbf{K m . 1 1}$ & San Rafael & Km. 11 & San Rafael & Km. 11 \\
\hline Gimnospermas & 1 & 0 & 1 & 0 & 1 & 0 \\
\hline Dicotiledóneas & 44 & 37 & 53 & 43 & 97 & 69 \\
\hline Monocotiledóneas & 13 & 9 & 18 & 17 & 29 & 22 \\
\hline Pteridofitas & 1 & 0 & 1 & 0 & 1 & 0 \\
\hline Licopodiáceas & 1 & 0 & 1 & 0 & 1 & 0 \\
\hline Total & $\mathbf{6 0}$ & $\mathbf{4 6}$ & $\mathbf{7 4}$ & $\mathbf{6 0}$ & $\mathbf{1 2 9}$ & $\mathbf{9 1}$ \\
\hline Especies nativas & & & & & 87 & 67 \\
\hline Especies introducidas & & & & & 32 & 23 \\
\hline Especies indeterminadas & & & & & 10 & 1 \\
\hline
\end{tabular}


milias con un número de géneros mayor a uno es del $26 \%$ y el porcentaje de familias con un solo género es el $74 \%$ de las familias encontradas.

En cuanto a la abundancia de especies encontradas en las dos comunidades, se encontraron tres especies con más de 100 individuos: la especie con mayor abundancia fue la yuca (Manihot esculenta Crantz), con 482 individuos colectados en todos los transectos de todas las chagras, seguido por la piña (Ananas comosus (L.) Merr.), con 209 individuos, y luego la coca (Erythroxylum coca Lam.), con 114 individuos. Con una abundancia superior a 50 individuos, se encontraron cinco especies: coca tingo María (Erythroxylum coca var. ipadu Plowman), el plátano, (Musa acuminata $\times$ balbisiana Colla) con 86 individuos cada uno, seguido del ñame (Dioscorea trifida L.f.) con 76 individuos. En el caso del Solanum sessiliflorum Dunal (lulo) se encontraron 63 individuos y para completar este grupo, está el dale dale, (Calathea allouia (Aubl.) Lindl.), con 52 individuos (Anexo 1).

Las chagras del Km. 11 presentan una menor diversidad florística, dado que la riqueza de una familia botánica se ve representada en la diversidad de géneros que posea. En total se registraron 129 especies en San Rafael, 53 de ellas compartidas con el $\mathrm{Km}$. 11 de Leticia, donde se encontraron un total de 91 especies. El 67,9\% de las especies encontradas son nativas de la región y el $24,5 \%$ son especies introducidas de diferentes regiones tropicales. El otro $7,3 \%$ solo han sido determinadas hasta género o familia pero es probable que un alto porcentaje de estos sean nativas.

Es importante destacar que aunque el número de especies compartidas es alto, los usos que se les asignan no son los mismos en ciertos casos.

La diversidad aquí registrada incluye algunas especies introducidas, desde el punto de vista botánico, pero desde el punto de vista cultural no son reconocidas como
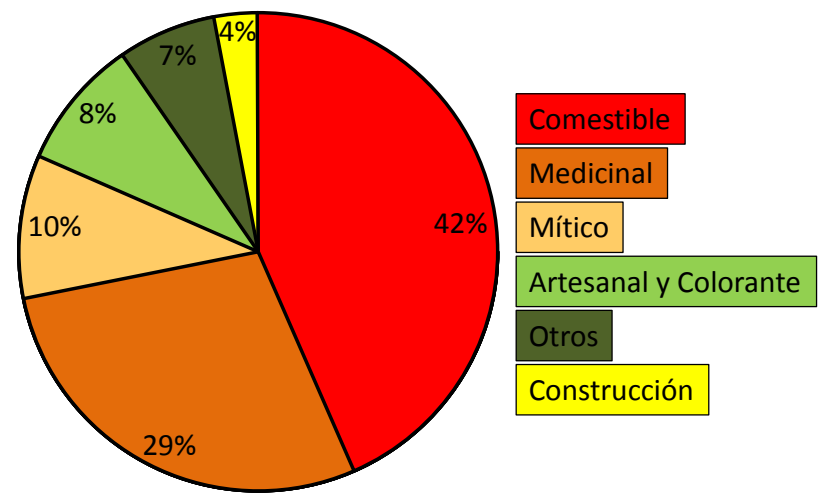

Figura 3. Categorías de uso de la comunidad de San Rafael, Colombia. foráneas. Un ejemplo de esto es el caso del plátano $(M$. acuminata $\times$ balbisiana). Aunque es una especie que no es nativa de la región amazónica, fue introducida hace siglos y representa, para las personas de la región, una planta con tradiciones más arraigadas que muchas plantas nativas de la región, que hasta ahora las están conociendo.

\section{Índices etnobotánicos}

\section{$\underline{\text { Valor de uso }}$}

Para las especies identificadas por las comunidades, se pudieron reconocer, según la clasificación etic realizada por de Marín-Corba et al. (2005) y Cárdenas et al. (2002), en la comunidad de San Rafael, seis categorías de uso (Figura 3): 1. Comestible, 2. Medicinal, 3. Mítico, 4. Construcción, 5. Artesanal y Colorante, 6. Tóxico y Otros (Siendo otros usos como Recreación, Ornamental, Alimento Animales). En la comunidad del Km. 11 de Leticia, se reconocieron siete categorías: (Figura 4) 1. Comestible, 2. Artesanal y Colorante, 3.Construcción, 4. Medicinal, 5. Mítico, 6. Tóxico y Otros, 7. Ventas (Ventas, tomado como sinónimo de Ingresos (Gausset 2004)). En esta comunidad, se encuentra un uso más, siendo uno de los más reconocidos en la comunidad del Km. 11 de Leticia, Ventas.

En la comunidad de San Rafael, las cosechas son utilizadas para el autoconsumo, ya que las ventas de sus cosechas no son significativas, no sólo porque no tienen un mercado al cual ofrecer su alimento (exceptuando a veces al Internado de San Rafael), sino porque también cada uno de los habitantes siembra y consume su propio alimento.

En el Km. 11 de Leticia se observa que los adultos mayores tienen mayor apego al conocimiento tradicional y especialmente en lo relacionado con plantas medicinales. Los datos aquí registrados en la categoría Medicinal fuer-

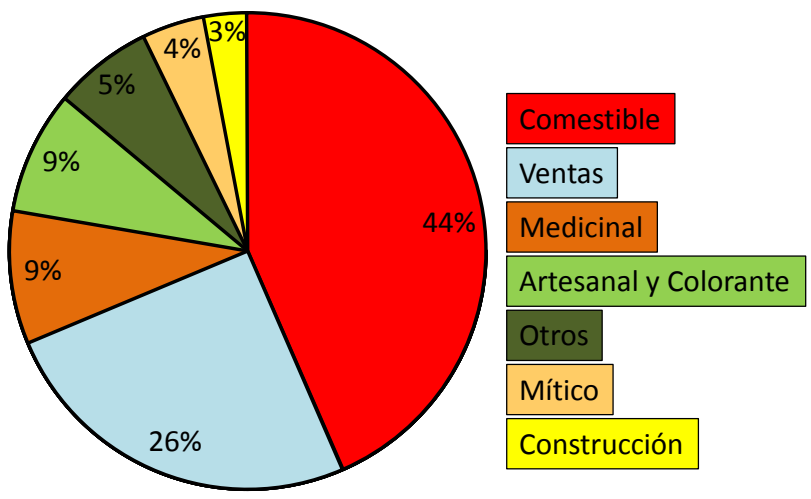

Figura 4. Categorías de uso de la comunidad Km. 11 de Leticia, Colombia. 


\section{Sierra \& Raz - Uso y Manejo de las Especies Sembradas en las Chagras de Dos Comunidades Murui-Muinane de la Amazonia Colombiana}

on proporcionados por este sector de la población. Algunos jóvenes conocen y utilizan especies medicinales pero sus prácticas son mezcladas con creencias adoptadas de otras culturas.

Tras realizar el procedimiento de sumatorio de usos, se realizaron intervalos de confianza para cada uno de dichos datos, y se encontraron para la comunidad de San Rafael que la mayoría de sus especies tenían un valor de uso (1) con un intervalo de confianza entre el $50,08 \%$ y el $66,47 \%$, seguidas por el valor de uso (2) con un intervalo de confianza entre el $21,25 \%$ y el $30,36 \%$. Las especies con un valor de uso igual a (3), oscilaban en un intervalo de confianza entre el $7,37 \%$ y el $18,53 \%$. En el $\mathrm{Km} .11$ de Leticia, se encontraron que la mayoría de las especies tenían un Valor de Uso (2) con un intervalo de confianza entre el $32,02 \%$ y el $52,43 \%$, seguido del valor de uso (1) con un intervalo de confianza entre el $28,82 \%$ y el $48,96 \%$. Con el valor de uso (3) los datos se ubican entre $10,8 \%$ y $26,98 \%$ (Figura 2 ).
Las especies que tienen un mayor valor de uso en la comunidad de San Rafael, son: yuca ( $M$. esculenta), maní (Arachis hypogaea L.), dale dale (C. allouia), ají (Capsicum chinense Jacq.), tabaco (Nicotiana tabacum L.) y umarí (Poraqueiba sericea Tul.), todas con las categorías de uso Alimenticia, Medicina y Mítica, más el almendro (Plukenetia volubilis L.) con las categorías de uso Alimenticia, Medicinal y Alimento para animales. Por otro lado, en la comunidad del Km.11 de Leticia, las especies con el mayor valor de uso: yuca (M. esculenta), ají (Capsicum sp. 1), limón (Citrus limon (L.) Osbeck) y granadilla (Passiflora sp. 1), todas con las mismas categorías de uso: Alimenticia, Medicina y Mítica.

\section{Índice de saliencia cultural}

Al aplicar el índice de saliencia cultural (frecuencia de especies nombradas como más importantes) se encontraron los siguientes valores (Tabla 2).

Tabla 2. Saliencia cultural comunidades San Rafael y Km. 11 de Leticia, Colombia.

\begin{tabular}{|c|c|c|c|}
\hline \multicolumn{2}{|l|}{ Nombres } & \multicolumn{2}{|c|}{ Saliencia en } \\
\hline Común & Científico & San Rafael & Km.11 de Leticia \\
\hline Acapú & Minquartia sp. 1 & 0,01 & \\
\hline Aji & Capsicum chinense Jacq. & 0,149 & 0,471 \\
\hline Ajingibre & Zingiberaceae sp. 1 & 0,02 & \\
\hline Albahaca & Ocimum cf. campechianum Mill. & & 0,014 \\
\hline Almendra & Terminalia cf. catappa L. & & 0,029 \\
\hline Araza & Eugenia stipitata McVaugh & 0,06 & 0,021 \\
\hline Arboldepan & Artocarpus sp. 1 & 0,02 & \\
\hline Asai & Euterpe precatoria Mart. & 0,104 & \\
\hline Bacaba & Oenocarpus bacaba Mart. & 0,04 & \\
\hline Batata & Ipomoea batatas (L.) Lam. & & 0,236 \\
\hline Caimo & Pouteria caimito (Ruiz \& Pav.) Radlk. & 0,12 & 0,25 \\
\hline Caña & Saccharum officinarum L. & 0,246 & 0,179 \\
\hline Chontaduro & Bactris gasipaes Kunth & 0,3 & 0,136 \\
\hline Chuchuguaza & Maytenus amazonica Mart. & 0,02 & \\
\hline Coca & Erythroxylum coca Lam. & 0,23 & 0,164 \\
\hline Copoazu & Theobroma grandiflorum (Willd. ex Spreng.) K.Schum. & 0,13 & 0,029 \\
\hline Daledale & Calathea allouia (Aubl.) Lindl. & & 0,043 \\
\hline Frijol & Phaseolus vulgaris $\mathrm{L}$. & & 0,021 \\
\hline Guamo & Inga spp. & 0,23 & 0,114 \\
\hline Hierbaluisa & Aloysia sp. 1 & 0,01 & \\
\hline \multirow[t]{2}{*}{ Huevitoro } & Theobroma sp. 1 & 0,03 & \\
\hline & Euphorbiaceae sp. 1 & & 0,029 \\
\hline Laurel & Spondias sp. 1 & 0,07 & \\
\hline Lulo & Solanum sessiliflorum Dunal & & 0,007 \\
\hline
\end{tabular}




\begin{tabular}{|c|c|c|c|}
\hline \multicolumn{2}{|l|}{ Nombres } & \multicolumn{2}{|c|}{ Saliencia en } \\
\hline Común & Científico & San Rafael & Km.11 de Leticia \\
\hline Macambo & Theobroma subincanum Mart. & 0,04 & \\
\hline Mafafa & Xanthosoma cf. sagittifolium (L.) Schott & & 0,057 \\
\hline Maíz & Zea mays L. & 0,08 & 0,064 \\
\hline Mango & Mangifera indica L. & 0,06 & \\
\hline Maní & Arachis cf. hypogaea L. & & 0,05 \\
\hline Maraca & Theobroma bicolor Humb. \& Bonpl. & 0,1 & 0,057 \\
\hline Marañon & Anacardium cf. occidentale L. & & 0,014 \\
\hline Milpesos & Oenocarpus bataua Mart. & 0,02 & \\
\hline Ñame & Dioscorea trifida L.f. & 0,353 & 0,364 \\
\hline Pimentón & Capsicum annuum L. & & 0,05 \\
\hline Piña & Ananas comosus (L.) Merr. & 0,411 & 0,386 \\
\hline Plátano & Musa acuminata $\times$ balbisiana Colla & 0,527 & 0,479 \\
\hline Sandia & Citrullus lanatus (Thunb.) Matsum. \& Nakai & & 0,036 \\
\hline Tabaco & Nicotiana tabacum L. & 0,07 & 0,114 \\
\hline Tomate & Solanum lycopersicum L. & & 0,043 \\
\hline Umari & Poraqueiba sericea Tul. & 0,33 & 0,186 \\
\hline Uva & Pouroma sp. 1 & 0,41 & 0,129 \\
\hline Yuca & Manihot esculenta Crantz & 0,83 & 0,793 \\
\hline
\end{tabular}

Para comparar las especies más nombradas como importantes con la abundancia encontrada en las chagras, se realizó una regresión lineal que determinó una correlación de 0,67 para la comunidad de San Rafael y 0,65 para la comunidad Km. 11 de Leticia (Figuras 5, 6).

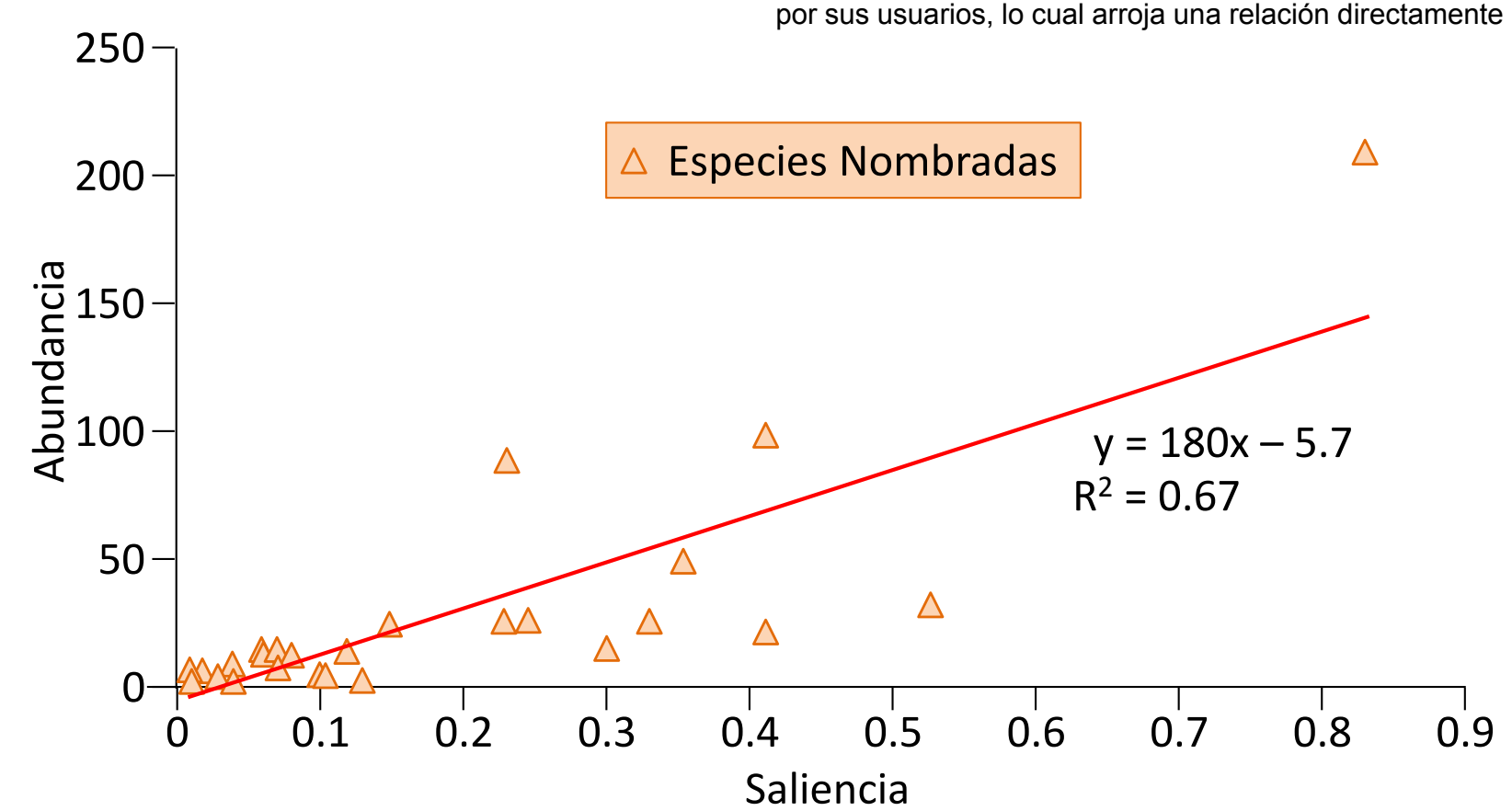

Figura 5. Valor de correlación entre la abundancia observada vs. la saliencia cultural de la comunidad de San Rafael, Colombia.
Este análisis muestra una relación casi directamente proporcional entre las especies nombradas y la abundancia encontrada. En el caso de las dos comunidades, la yuca (M. esculenta) es la especie que se encuentra con mayor frecuencia en las chagras y es también la más nombrada por sus usuarios, lo cual arroja una relación directamente 


\section{Sierra \& Raz - Uso y Manejo de las Especies Sembradas en las Chagras de Dos Comunidades Murui-Muinane de la Amazonia Colombiana}

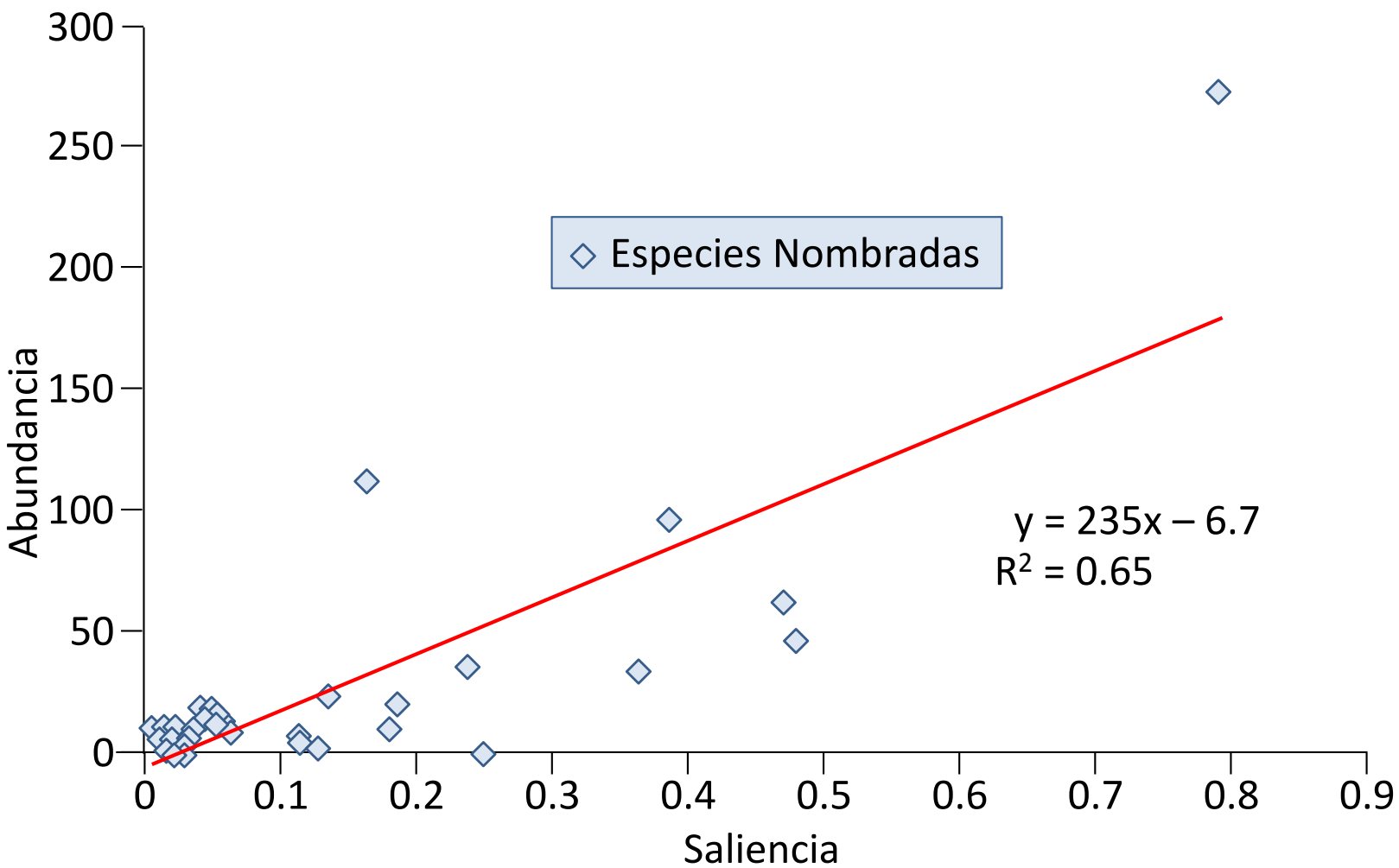

Figura 6. Valor de correlación entre la abundancia observada vs. la saliencia cultural de la comunidad Km. 11 de Leticia, Colombia.

proporcional. Sin embargo se observan ciertas especies en las que la diferencia entre saliencia y abundancia es muy notoria, como es el caso del acapú (Minquartia sp. 1) y de la chuchuguaza (Maytenus cf. amazonica Mart.) cuya saliencia no se ve representada en la abundancia de estas especies en la chagra. Ambas son especies que normalmente se encuentran en estado silvestre y que al realizar la chagra, se mantuvieron. Existe un caso atípico con el caimito (Pouteria caimito (Ruiz \& Pav.) Radlk.) especie que es muy nombrada (Saliencia de 0.2) y que sin embargo sólo fue colectada en una chagra. Aunque esta especie normalmente se encuentra en estado silvestre, se observa casos de semi-domesticación, ya que están intentando sembrarla por el interés en sus frutos, para poder venderlo con más facilidad en el mercado de Leticia. Caso contrario se observa con la piña ( $A$. comosus) y la coca ( $E$. coca) especies que presentan una muy alta abundancia en las chagras, pero cuyo nivel de saliencia cultural no es tan alto como sería de esperar si se toma en cuenta que son especies que se encuentran en casi todas las chagras. Aunque estas especies son reconocidas, muchas veces son especies tan obvias en el pensamiento colectivo, que ni siquiera se mencionan y pueden parecerse subvaloradas.
Índice modificado de Valor de Importancia de Byg \& Balslev

El índice modificado de Byg \& Balslev indica que la especie que tuvo mayor consenso dentro de la comunidad de San Rafael fue la yuca (M. esculenta), reconocida por el $100 \%$ de las personas como la más sembrada. En la comunidad del Km. 11 de Leticia, la especie con mayor consenso es también $M$. esculenta. Esta especie se cultiva en las dos comunidades, en todas las chagras. Adicionalmente, la yuca es la especie que más nombres comunes posee.

La piña ( $A$. comosus) es también una especie nombrada en todas las chagras, sin embargo en una de ellas no fue vista: ni durante el recorrido etnobotánico, ni en los levantamientos realizados. La piña, al igual que la yuca, es una especie ampliamente cultivada y conocida con muchos nombres, lo que indica un rango de importancia por parte de la comunidad, que la ubica en el nivel alto en ambas comunidades.

Tanto en la comunidad de San Rafael como en la comunidad del Km. 11 de Leticia, el primer lugar en el índice de valor de importancia es para la yuca (M. esculenta). En el segundo lugar (nivel muy alto), en la comunidad de San Rafael, figura el ají ( $C$. cf. chínense), utilizado con fines comestibles, medicinales y míticos. En la comunidad del 
Km. 11 de Leticia, el segundo lugar es ocupado por la uva caimarona (Pourouma cecropiifolia Mart.), destacado por sus frutos comestibles, aunque de esta misma especie también se utilizan las hojas para sacar la ceniza necesaria para la elaboración del "mambe." Cuando se refiere al uso de las hojas de esta especie, el nombre común "yarumo" es aplicado. Las hojas de especies de Cecropia (el género tradicionalmente asociado con la palabra "yarumo") también son utilizadas para preparar el mambe (polvo de coca macerado con cenizas de yarumo, fundamental en la cultura Murui) en el Km. 11, pero las especies de Cecropia son silvestres y no sembradas. Otras especies nombradas dentro de la categoría muy alta en las dos comunidades indígenas son: el plátano ( $M$. acuminata $\times$ balbisiana), la piña ( $A$. comosus), y el ñame $(D$. trifida). Estas especies son las más sembradas, no sólo por la facilidad de su cultivo sino también por ser consideradas las de mayor valor alimenticio dentro de los sembradíos. En la categoría con consenso alto se encuentran especies como el caimito ( $P$. caimito), la coca ( $E$. coca) y la guama (Inga spp.) Dentro de las especies importantes en la comunidad de San Rafael, cabe resaltar el tabaco (N. tabacum) que es usado por la mayoría de la comunidad con propósitos míticos y medicinales. En cambio, en la comunidad del Km. 11 de Leticia, el tabaco se ubica en la categoría baja, ya que en las entrevistas fue nombrado sólo por una persona. A pesar de ser muy usado y de encontrarse sembrado en varias partes, los miembros de la comunidad no lo consideran tan importante como otras especies; los hombres lo reconocen, pero le dieron prioridad en la entrevista a otras especies; las mujeres no lo reconocen como parte fundamental de la cultura Murui, ya que sus usuarios se reducen a los abuelos y los curacas o jefes de la comunidad. El tabaco se ha ido dejando de sembrar debido al fácil acceso a cigarrillos o tabaco procesado en el comercio de Leticia.

El ají (C. chinense) en la comunidad del Km. 11 de Leticia, se ubica en el nivel medio. Aunque es sembrado en cierta medida, no fue muy nombrado en las entrevistas, lo cual indica que no se lo considera una especie con importancia alta, o que por ser una especie tan sembrada, no suele asociarse como especie importante porque ya es parte de un común. Lo mismo sucede con $P$. cecropiifolia en la comunidad de San Rafael; aunque la hoja de esta especie se utiliza esporádicamente para la preparación del mambe, es mucho más común el uso de Cecropia spp. para la elaboración del mismo.

El barbasco (Clibadium surinamense L.) se ubica en la comunidad de San Rafael en el nivel medio, ya que es utilizado para la pesca, que en esta comunidad es una actividad reconocida y altamente practicada. Por otro lado, en la comunidad del Km. 11, esta especie se ubica en la categoría baja. Allí la pesca es una práctica poco realizada, ya que la cercanía a Leticia facilita la consecución de pescado en el mercado de la ciudad.

\section{Discusión}

\section{Estudio florístico}

En la comunidad del Km. 11 la siembra de especies en las chagras se limita muchas veces a ciertos productos, los cuales son en su mayor parte los que tienen más consumo en el casco urbano de Leticia. Estas especies son en su mayoría frutales traídos de otros lugares de la Amazonia y que no son propios de su cultura, tales como el copoazú (Theobroma bicolor Humb. \& Bonpl.), el arazá (Eugenia stipitata McVaugh) y demás frutales a los que tienen fácil acceso.

La yuca es el alimento más importante, el más vendido y uno de los cultivos de producción más rápida. En varias chagras de la comunidad de Leticia, se destina la mayor parte del terreno a la siembra casi exclusiva de la yuca brava ( $M$. esculenta), ya que esta variedad no puede ser robada con tanta facilidad y el riesgo de pérdida de la cosecha es menor comparado con otras variedades de yuca. La chagra es un espacio abierto sin una cerca u otra barrera física y el robo se presenta por parte de personas que no tienen chagras, o las tienen descuidadas (con malas cosechas o baja producción). La yuca brava, una variedad de yuca con niveles de toxicidad más altos que otras variedades, es utilizada para realizar la fariña y el cazabe (alimentos típicos de la región), pero necesita un proceso de elaboración muy largo, por lo tanto, el riesgo de robo es mínimo. Datos similares fueron observados por Peña-Vanegas et al. (2009) y Arbelaez \& Martínez (2012).

Las especies registradas entre las más abundantes en este estudio también fueron reportadas como abundantes por Henao (1990), en la comunidad Uitoto de Chorrera, Putumayo, y Almanza (1993), en el Caquetá. Estos autores además encontraron en las chagras muchas especies de lento crecimiento como frutales, confirmando lo encontrado por Vélez y Vélez (1999), quienes consideraron que los Uitotos y Bora en la Amazonia son más sedentarios comparadas con otras etnias que tienden a sembrar una mayor proporción de especies de rápido crecimiento. En este estudio los frutales son más abundantes en Km. 11 comparado con San Rafael, pero esto no es una función de la tendencia de los habitantes a permanecer más tiempo en sus comunidades, sino es relacionado con la venta de frutos en los mercados de Leticia.

A pesar de ser un cultivo no típicamente asociado con la Amazonia, la siembra del maíz (Zea mays L.) fue importante en esta región en el pasado (Morcote-Ríos \& León Sicard 2012), y estaba ligada a la tradición cultural y a un trasfondo mítico. Hoy en día, se usa más que todo como alimento para las aves de corral, tanto en Leticia como en San Rafael. En este punto también se ven similitudes con lo encontrado por Peña-Vanegas et al. (2009). 


\section{Sierra \& Raz - Uso y Manejo de las Especies Sembradas en las Chagras de Dos Comunidades Murui-Muinane de la Amazonia Colombiana}

Índices etnobotánicos

Valor de Uso

Los valores y categorías de uso encontrados en la comunidad de San Rafael y en la comunidad del Km. 11 de Le- ticia son comparables con las descritas por otros autores que han trabajado en la región (Tabla 3). Cárdenas et al. (2002), en la comunidad de Lagarto Cocha, encontraron categorías de uso que concuerdan con los datos registrados aquí, como es el caso del uso Medicinal ( $31 \%$ en la comunidad de Lagarto Cocha y $29 \%$ en la comunidad de

Tabla 3. Índice Modificado de Byg \& Balslev (2001) comunidades San Rafael y Km. 11 de Leticia, Colombia. Sturges: 1. Nivel mu alto; 2 . Nivel alto; 3 . Nivel medio; 4 . Nivel bajo; y 5 . Ninguno.

\begin{tabular}{|c|c|c|c|c|c|}
\hline \multicolumn{2}{|l|}{ Nombres } & \multicolumn{2}{|c|}{ Índices } & \multicolumn{2}{|c|}{ Sturges class intervals } \\
\hline Científico & Común & San Rafael & $\begin{array}{c}\mathrm{Km} .11 \text { de } \\
\text { Leticia }\end{array}$ & San Rafael & $\begin{array}{c}\text { Km. } 11 \text { de } \\
\text { Leticia }\end{array}$ \\
\hline Manihot esculenta Crantz & Yuca & 100 & 100 & 1 & 1 \\
\hline Musa acuminata $\times$ balbisiana Colla & Plátano & 61,5 & 72,7 & 2 & 2 \\
\hline Dioscorea trifida L.f. & Ñame & 69,2 & 63,6 & 1 & 2 \\
\hline Ananas comosus (L.) Merr. & Piña & 53,8 & 63,6 & 2 & 2 \\
\hline Capsicum chinense Jacq. & $A j i$ & 92,3 & 18,2 & 1 & 3 \\
\hline Pourouma cecropiifolia Mart. & Uva & 15,4 & 90,9 & 3 & 1 \\
\hline Pouteria caimito (Ruiz \& Pav.) Radlk. & Caimo & 53,8 & 45,5 & 2 & 2 \\
\hline Bactris gasipaes Kunth & Chontaduro & 30,8 & 63,6 & 3 & 2 \\
\hline Erythroxylum coca Lam. & Coca & 53,8 & 36,4 & 2 & 3 \\
\hline Inga spp. & Guamo & 30,8 & 54,5 & 3 & 2 \\
\hline Poraqueiba sericea Tul. & Umari & 30,8 & 45,5 & 3 & 2 \\
\hline Saccharum officinarum L. & Caña & 23,1 & 45,5 & 3 & 2 \\
\hline Ipomoea batatas (L.) Lam. & Batata & 53,8 & 9,1 & 2 & 4 \\
\hline Nicotiana tabacum L. & Tabaco & 46,2 & 9,1 & 2 & 4 \\
\hline $\begin{array}{l}\text { Theobroma grandiflorum (Willd. } \\
\text { ex Spreng.) K.Schum. }\end{array}$ & Copoazu & & 45,5 & 5 & 2 \\
\hline Theobroma bicolor Humb. \& Bonpl. & Maraca & 15,4 & 27,3 & 3 & 3 \\
\hline Calathea allouia (Aubl.) Lindl. & Daledale & 23,1 & 18,2 & 3 & 3 \\
\hline Xanthosoma cf. sagittifolium (L.) Schott & Mafafa & 38,5 & & 2 & 5 \\
\hline Eugenia stipitata McVaugh & Araza & & 27,3 & 5 & 3 \\
\hline Euterpe precatoria Mart. & Asai & & 27,3 & 5 & 3 \\
\hline Zea mays L. & Maíz & 7,7 & 18,2 & 4 & 3 \\
\hline Clibadium surinamense L. & Barbasco & 15,4 & 9,1 & 3 & 4 \\
\hline Mangifera indica L. & Mango & & 18,2 & 5 & 3 \\
\hline Anacardium occidentale L. & Marañon & 7,7 & 9,1 & 4 & 4 \\
\hline Maytenus amazonica Mart. & Chuchuguaza & & 9,1 & 5 & 4 \\
\hline Oenocarpus bacaba Mart. & Bacaba & & 9,1 & 5 & 4 \\
\hline Arachis cf. hypogaea L. & Maní & 7,7 & & 4 & 5 \\
\hline Capsicum annuum L. & Pimentón & 7,7 & & 4 & 5 \\
\hline Citrullus lanatus (Thunb.) Matsum. \& Nakai & Sandia & 7,7 & & 4 & 5 \\
\hline Euphorbiaceae sp. 1 & Sachalnchi & 7,7 & & 4 & 5 \\
\hline Ocimum cf. campechianum Mill. & Albahaca & 7,7 & & 4 & 5 \\
\hline Phaseolus vulgaris $\mathrm{L}$. & Frijol & 7,7 & & 4 & 5 \\
\hline
\end{tabular}




\begin{tabular}{|l|l|c|c|c|c|}
\hline Nombres & \multicolumn{2}{c|}{ Índices } & \multicolumn{2}{c|}{ Sturges class intervals } \\
\hline Científico & Común & San Rafael & $\begin{array}{c}\text { Km. 11 de } \\
\text { Leticia }\end{array}$ & San Rafael & $\begin{array}{c}\text { Km. 11 de } \\
\text { Leticia }\end{array}$ \\
\hline Solanum lycopersicum L. & Tomate & 7,7 & & 4 & 5 \\
\hline Solanum sessiliflorum Dunal & Lulo & 7,7 & & 4 & 5 \\
\hline Terminalia cf. catappa L. & Almendra & 7,7 & & 4 & 5 \\
\hline
\end{tabular}

San Rafael); en los estudios de Hammond et al. (1995), Marín-Corba et al. (2005) y Sierra (2007), en la categoría Alimenticio o Comestible, se encontraron porcentajes superiores al 40\% (Tabla 4). El uso Mítico, Ilamado "Cultural" en Marín-Corba et al. (2005), tiene un porcentaje similar en Km. 11 y San Rafael (7\%), al igual que el encontrado en Sierra (2007). El valor de uso encontrado en Leticia para la categoría Ventas, es de un $26 \%$, valor similar al encontrado por Hammond et al. (1995) que fue del $22 \%$.

Es importante resaltar que aunque las comidas provienen de una misma especie, los usos y maneras de preparación varían de una comunidad a otra. Un ejemplo de esto se observa en el uso de la yuca (M. esculenta), de la cual se saca la fariña o el casabe, alimento de uso diario en toda la Amazonia. Por otra parte el caldo con hojas de yuca, las sopas y el caldo de yuca dulce, son preparaciones casi exclusivas de las comunidades interiores como San Rafael y prácticamente desconocidas en Leticia. Estas observaciones concuerdan con lo encontrado por Peña-Vanegas et al. (2009).

\section{Influencia de factores culturales y históricos}

San Rafael: Las respectivas bonanzas del caucho y la coca generaron desinterés en la chagra. En la época de las caucherías, las chagras fueron abandonadas porque las comunidades fueron obligadas a trabajar, mientras que en el auge de la coca, les resultaba mucho más rentable trabajar como raspachines (los que procesan la coca en cocaína). Aunque la bonanza del caucho fue hace más de 80 años, las respuestas demuestran que las comunidades indígenas tienen aún en su memoria como capítulo reciente ese hecho. Una influencia más reciente en la diversidad de las chagras es la presencia de una base armada en el municipio de El Encanto, donde llegan embarcaciones con alimento "blanco", una categoría que incluye la papaya, el lulo y el marañón, especies nuevas en la región. Estas especies se adaptaron a los suelos locales y crecieron con facilidad, sustituyendo a otras especies nativas de la zona. Otros ejemplos incluyen la sustitución del mango por el dale dale, el plátano por los ñames y los tubérculos nativos. En algunos casos lo que

Tabla 4. Comparación de categorías de uso por comunidades. ${ }^{1}$ Cárdenas et al. 2002; ${ }^{2} \mathrm{Hammond}$ et al. 1995; ${ }^{3}$ Sierra 2007.

\begin{tabular}{|l|c|c|c|c|c|c|}
\hline \multirow{2}{*}{ Categorías de uso } & \multicolumn{7}{|c|}{ Comunidades } \\
\cline { 2 - 7 } & $\begin{array}{c}\text { Lagarto } \\
\text { Cocha }^{1}\end{array}$ & Churumbelo $^{\mathbf{2}}$ & $\begin{array}{c}\text { Las } \\
\text { Palmeras }^{2}\end{array}$ & $\begin{array}{c}\text { Monilla } \\
\text { Amena }^{3}\end{array}$ & San Rafael & $\begin{array}{c}\text { Km. 11 de } \\
\text { Leticia }\end{array}$ \\
\hline ALIMENTO/COMESTIBLE & $22 \%$ & $16 \%$ & $50 \%$ & $52 \%$ & $42 \%$ & $44 \%$ \\
\hline ARTESANAL & $8 \%$ & $3 \%$ & $2 \%$ & $1 \%$ & $7 \%$ & $9 \%$ \\
\hline ASERRíO & $12 \%$ & $26 \%$ & $0 \%$ & $0 \%$ & $0 \%$ & $0 \%$ \\
\hline COLORANTES & $1 \%$ & $1 \%$ & $0 \%$ & $0 \%$ & $0 \%$ & $0 \%$ \\
\hline COMBUSTIBLES & $3 \%$ & $15 \%$ & $0 \%$ & $0 \%$ & $0 \%$ & $0 \%$ \\
\hline CONSTRUCCIÓN & $7 \%$ & $6 \%$ & $13 \%$ & $0 \%$ & $4 \%$ & $3 \%$ \\
\hline CULTURAL/ MÍTICO & $6 \%$ & $3 \%$ & $0 \%$ & $7 \%$ & $10 \%$ & $4 \%$ \\
\hline FORRAJE & $0 \%$ & $2 \%$ & $0 \%$ & $0 \%$ & $0 \%$ & $0 \%$ \\
\hline MEDICINAL & $31 \%$ & $20 \%$ & $10 \%$ & $13 \%$ & $29 \%$ & $9 \%$ \\
\hline ORNAMENTAL & $5 \%$ & $6 \%$ & $0 \%$ & $11 \%$ & $0 \%$ & $0 \%$ \\
\hline PSICOTRÓPICO & $2 \%$ & $0 \%$ & $0 \%$ & $0 \%$ & $0 \%$ & $0 \%$ \\
\hline TÓXICO/VENENO & $3 \%$ & $1 \%$ & $0 \%$ & $1 \%$ & $0 \%$ & $0 \%$ \\
\hline OTROS & $3 \%$ & $3 \%$ & $3 \%$ & $14 \%$ & $8 \%$ & $5 \%$ \\
\hline VENTAS & $0 \%$ & $0 \%$ & $22 \%$ & $0 \%$ & $0 \%$ & $26 \%$ \\
\hline TOTAL & $100 \%$ & $100 \%$ & $100 \%$ & $100 \%$ & $100 \%$ & $100 \%$ \\
\hline
\end{tabular}




\section{Sierra \& Raz - Uso y Manejo de las Especies Sembradas en las Chagras de Dos Comunidades Murui-Muinane de la Amazonia Colombiana}

se perdió no fueron las especies sino los usos. Las entrevistas resaltan que se han perdido mucho más los usos de sus especies nativas, que las especies como tal. Las especies encontradas hace años eran asociadas a mitos y creencias que explicaban la presencia de dichas especies en sus chagras (por ejemplo el mito de "Monilla Amena" como el árbol de la cosecha de la cual salían todas las especies de una chagra).

Aunque la importancia de los cultivos como fuente de ingresos en San Rafael es menor que en el Km. 11, ahora las especies encontradas en las chagras son dadas más por lo que pueda ofertarse en los intercambios con las embarcaciones, intercambio con los vecinos, o intercambio directamente con el internado. Es posible que dentro de una o dos generaciones, el comercio se aumentará y los patrones de uso y diversidad de las especies aquí reportados se cambiarán: una transculturación acelerada no por la cercanía a un centro urbano, sino por el aumento de la población militar en la zona.

En el caso de las especies más nombradas, yuca, ají y piña, los datos son similares y comparables a los encontrados por van der Hammen (1992) en comunidades de la cultura Yucuna. Estas especies son reconocidas por tener muchas variedades sembradas en las chagras (cultivariedades), y también por ser especies con transfondo mítico que afirman proteger otras especies y sirven para delimitar la chagra (van der Hammen 1992). En el caso de Manihot esculenta, las hojas son utilizadas como complemento alimenticio, son cocinadas y mezcladas con otros alimentos, sirviendo como "hojas o vegetales", un uso también encontrado por Brandão \& Brandão (1991), y por Giraldo et al. (2006), quienes reportan que las hojas sirven como suplementos alimenticios para niños de Brasil.

En cuanto a la ubicación de las especies encontradas en las chagras, al igual que lo encontrado por van der Hammen (1992) y Vélez y Vélez (1999), el sembradío de la coca y el tabaco, se considera el corazón de la chagra, por eso se ubica en el centro y los demás cultivos se distribuyen alrededor de este.

Km. 11 de Leticia: Para esta comunidad, las especies medicinales, y algunas alimenticias como el caso del ají (Capsicum spp.) y del tabaco (N. tabacum) se han dejado de usar, bien sea porque no se han sembrado hace mucho tiempo (es decir, porque se perdió la semilla), o porque el SINCHI (Instituto Amazónico de Investigaciones Científicas, una entidad del estado colombiano) los provee con semillas de nuevas variedades alimenticias, lo que para ellos significa una mayor diversidad. En el caso del ají y el tabaco, el fácil acceso a la ciudad hace que las personas de esta comunidad dejen de sembrar estas especies y compren productos manufacturados (salsa de ají, cigarrillos). Para los miembros de la Km. 11, todas las especies que siembran son importantes, simplemente por el hecho de sembrarlas, pero no destacan ninguna en particular. En el caso de la yuca reconocen que han prescindido de las variedades de más lento crecimiento, dando prioridad en la siembra a las variedades que demoran menos de un año en producir. Estas prioridades son dadas por la facilidad de alimentación, ya que pueden conseguir alimentos similares en Leticia, por la falta de espacios para sembrar especies que demoran años, e incluso en ciertos casos, por la pereza que se genera en las personas, inducida por la rapidez de obtención y facilidad de consumo de especies inmediatas. La comunidad del Km. 11 de Leticia tiene una ubicación estratégica para la consecución de alimentos variados que se pueden encontrar en tierras amazónicas, sin la necesidad de realizar una chagra, una observación también realizada por Arbeláez y Martínez (2012) en la misma comunidad.

El énfasis hoy día en ventas en el Km. 11 puede ser explicado por la cercanía de esta comunidad a los mercados de Leticia, pero los procesos de transculturación empezaron mucho antes, en la época de la explotación del caucho. El desplazamiento forzado de las comunidades Muinani seguramente es un factor importante en su historia de transculturación (y de su resiliencia). La transculturación de la comunidad del Km. 11 se ve en fenómenos como el aumento en el uso de productos prefabricados, preferencia para cultivar productos rentables, (incluyendo la venta del mambé a gente fuera de la comunidad), la disminución en el uso de plantas medicinales y el reducido interés por parte de los jóvenes en rituales y prácticas tradicionales asociados a la siembra y cosecha.

\section{Conclusiones}

En la comunidad de San Rafael y en la del Km. 11 de Leticia, sus habitantes identifican las especies de sus chagras, conocen sus usos, saben cómo cultivarlas y qué tipo de preparaciones se pueden hacer con ellas. Sin embargo el porcentaje de la población que practica la agricultura tradicional es menor en Km. 11 que en San Rafael. En San Rafael se observa una diversidad de especies más alta de la que se puede encontrar en la comunidad del Km. 11 de Leticia. Respecto a los usos, se pudo observar que en San Rafael estos siguen siendo los tradicionales: alimentación, medicinal y mítico, mientras que la producción comercial es mucho más importante en $\mathrm{Km}$. 11.

Se confirma que las especies más sembradas en las chagras de ambas comunidades son: la yuca (M. esculenta), seguida por la piña (A. comosus) y el plátano (M. acuminata $\times$ balbisiana). Estas especies también son las que mayor cantidad de usos tienen y las más vendidas.

Para especies con un componente ritual dentro de la cultura, como la coca (E. coca), en San Rafael, siguen siendo consideradas como especies místicas. En el Km. 11 de 
Leticia, en cambio, han llegado incluso a venderla a las personas ajenas a la comunidad con fines comerciales, desvirtuando el carácter sagrado que tiene la coca dentro de las comunidades indígenas.

En cuanto a las creencias, que incluyen tiempos y lugares apropiados para la siembra, quiénes deben o no sembrar, mantener y cosechar cada especie y practicar los rituales para obtener mejores resultados en las cosechas, hay mayor observancia de ellas en la comunidad de San Rafael. En el Km. 11 de Leticia se observa que los adultos mayores tienen mayor conocimiento sobre el manejo de sus chagras, y que algunos jóvenes conocen y utilizan las especies pero su conocimiento está mezclado con creencias adoptadas de otras culturas cercanas a Leticia, como los Ticunas o comunidades Brasileñas.

Resaltamos que, después de la producción de alimentos para el autoconsumo, en San Rafael el cultivo de especies para uso medicinal es la segunda categoría más importante, mientras que la siembra de plantas (alimenticias o no) con propósito comercial prevalece en la comunidad $\mathrm{Km} .11$ de Leticia. Esta diferencia se debe más que todo, al fácil acceso que la comunidad de $\mathrm{Km}$. 11 tiene a mercados para vender sus productos. La facilidad de acceso a servicios de salud (clínicas, hospitales) en Leticia versus San Rafael también puede ser un factor que explica las diferencias observadas.

Concluimos que la distancia es un factor que acelera la transculturación, pero no es el único y la comunidad de San Rafael no es inmune. Como se ha mencionado, la población militar de El Encanto está aumentando y valdría la pena repetir este estudio en 10 o 20 años.

\section{Literatura Citada}

Albuquerque U.P., R.F.P. Lucena \& L. Alencar. 2010. Métodos e técnicas para a coleta de dados etnobiologicos. Pp. 39-63 en Métodos e Técnicas na Pesquisa Etnobiologica e Etnoecologica. Editado por U.P. Albuquerque \& R.F.P. Lucena. Nupeea, Recife, Brazil.

Almanza, M. 1993. La Biodiversidad en la Amazonia Colombiana: A través de las diferentes formas de uso del espacio. Centro de Documentación, Instituto Amazónico de Investigaciones Científicas (SINCHI), Leticia, Colombia.

Arbeláez, J. \& L. Martínez. 2012. Diagnostico Socio Agroforestal para la Diversificación de Chagras y Fortalecimiento de la Seguridad Alimentaria en el Resguardo Indígena Km 6 y 11, Parcialidad Comunidad Km 11, n+maira $n a+m e k+i b+r+($ Patio de Ciencia Dulce), Municipio de Leticia, Departamento de Amazonas. Trabajo de grado. Especialista de Gestión de Proyectos. Universidad Nacional Abierta y a Distancia (UNAD), Bogotá, Colombia.
Bernal, R., G. Galeano, Z. Cordero, P. Cruz, M. Gutiérrez, A. Rodríguez \& H. Sarmiento. 2006. Diccionario de Nombres Comunes de las Plantas de Colombia. Versión en línea. Instituto de Ciencias Naturales, Universidad Nacional de Colombia, Bogotá, Colombia. www.biovirtual.unal. edu.co/diccionario/

Bonilla, E. \& P. Rodríguez. 2005. Más allá del Dilema de los Métodos: La investigación en ciencias sociales. Tercera Edición. Norma, Bogotá, Colombia.

Boom, B.M. 1990. Useful plants of the Panare Indians of the Venezuelan Guayana. Advances in Economic Botany 8:57-65.

Borgatii, S. \& M. Natick. 1996. Anthropac 4.0. Analytic Technologies, Columbia, South Carolina, U.S.A.

Brandão, C. \& F. Brandão. 1991. Alternative Nourishment: An educational program on nutrition based on regional solutions and simplified technology. National Conference of Bishops of Brazil (CNBB), Brazil.

Brummitt, K. \& C. Powell. 1992. Authors of Plant Names: A list of authors of scientific names of plants with recommended standard forms of their names, including abbreviations. Royal Botanic Gardens, Kew, U.K.

Byg, A. \& H. Balslev. 2001. Diversity and uses of palms in Zahamena, eastern Madagascar. Biodiversity and Conservation 10:951-970. dx.doi.org/10.1023/A:1016640713643

Cárdenas, D., C. Marín, S. Suárez, C. Guerrero \& P. Nofuya. 2002. Plantas Útiles en Dos Comunidades del Departamento del Putumayo. Instituto Amazónico de Investigaciones Científicas (SINCHI), Leticia, Colombia.

Castro-S., H. \& S. Galán-R. 2003. Conocimiento y manejo del bosque. Los Uitotos en la Amazonia colombiana. Biodiversidad 38:14-16. www.grain.org/e/993

CIFISAM. 2005. La Chagra: Un espacio de roles, aprendizajes y autoabastecimiento. Editorial CÓDICE. Primera edición. San Vicente del Caguán, Caquetá, Colombia.

CORPOAMAZONÍA (Corporación para el Desarrollo Sostenible del Sur de la Amazonia). 2008. Informe de Gestión y Financiero de Corpoamazonía. CORPOAMAZONÍA, Puerto Leguizamo, Putumayo, Colombia.

Cunningham, A. 2001. Etnobotánica aplicada. Pueblos, usos de plantas silvestres y conservación. Pueblos y Plantas 4. Manual de conservación. WWF, UNESCO, and Royal Botanic Gardens, Kew, U.K.

Galán, S. 2003. Manejo y Enriquecimiento del Bosque a Través del Uso de los Frutales Cultivados en las Chagras y Rastrojos de un Núcleo Familiar Indígena de Araracu- 


\section{Sierra \& Raz - Uso y Manejo de las Especies Sembradas en las Chagras de Dos Comunidades Murui-Muinane de la Amazonia Colombiana}

ara, Medio Caquetá, Colombia. Tesis Ecología. Pontificia Universidad Javeriana, Bogotá, Colombia.

Galeano, G. 2000. Forest use at the Pacific Coast of Chocó, Colombia: A quantitative approach. Economic Botany 54(3):358-376. dx.doi.org/10.1007/BF02864787

Gasca A., H.J. 2005. El significado de los escarabajos (Coleoptera: Scarabaeoidea) en una comunidad Uitoto de Leticia, Amazonas (Colombia): Una exploración preliminar a su conocimiento etnoentomológico. Boletín Sociedad Entomológica Aragonesa 36:309-315. www.seaentomologia.org/PDF/BOLETIN 36/B36-067-309.pdf

Gausset, Q. 2004. Ranking local tree needs and priorities through an interdisciplinary action research approach. The Journal of Transdisciplinary Environmental Studies 3(1):1-17. www.journal-tes.dk/vol\%203\%20no\%201/ Quentin\%20Gausset lav.pdf?id=00029

Gentry, A. 1993. A Field Guide to the Families and Genera of Woody Plants of Northwest South America. University of Chicago Press, Chicago, Illinois, U.S.A.

Giraldo, A., R. Velazco \& J. Aristizabal. 2006. Obtención de harina a partir de hojas de yuca (Manihot esculenta Crantz) para consumo humano. Revista de Biotecnología en el Sector Agropecuario y Agroindustrial 4(1):33-42. www.unicauca.edu.co/biotecnologia/ediciones/vol4/4.pdf

Hammond, D.S., P.M. Dolman \& A.R. Watkinson. 1995. Modern Ticuna swidden-fallow management in the Colombian Amazon: Ecologically integrating market strategies and subsistence-driven economies? Human Ecology 23:335-356. dx.doi.org/10.1007/BF01190136

Henao, C.I. 1990. Interpretación Etnobotánica del Mito del "Árbol de las Frutas" en la Tradición Oral Huitoto como Modelo de la Domesticación de las Plantas en La Chorrera Amazonas. Tesis de Biología, Pontificia Universidad Javeriana, Bogotá, Colombia.

Hoffman, B. \& T. Gallaher. 2007. Importance indices in ethnobotany. Ethnobotany Research \& Applications 5:201-218. http://lib-ojs3.lib.sfu.ca:8114/index.php/era/ article/view/130

Jiménez Escobar, N.D. 2012. Uso y Conocimiento de Árboles en la Comunidad Campesina de la Bahía de Cispatá, Departamento de Córdoba-Colombia. Tesis de Maestría en Ciencias, Biología. Universidad Nacional de Colombia, Bogotá, Colombia.

Jiménez Escobar, N.D., C. Estupiñán, N. Sánchez \& C. Garzón. 2009. Etnobotánica de la media montaña de la Serranía del Perijá. Pp. 393-416 en Colombia Diversidad Biótica (VIII): Media y baja montaña de la Serranía del Perijá. Editado por J.O. Rangel-Ch. Universidad Nacional de Colombia, Instituto de Ciencias Naturales, CORPOCESAR - REVIVE, Bogotá, Colombia.

Jiménez Escobar, N.D., U.P. Albuquerque \& J.O. RangelCh. 2011. Huertos familiares en la Bahía de Cispatá, Cordobá, Colombia. Bonplandia 20 (2):309-328. http://ibone. unne.edu.ar/objetos/uploads/documentos/bonplandia/ public/20 2/309 328.pdf

Karremans, J. 1994. Sociología para el Desarrollo, Métodos de Investigación y Técnicas de la Entrevista. Serie Técnica, Informe Técnico $\mathrm{N}^{\circ} 228$. Centro Agronómico Tropical de Investigación y Enseñanza (CATIE), Turrialba, Costa Rica.

Lamont, S.R., W.H. Eshbaugh \& A.M. Greenberg. 1999. Species composition, diversity, and use of homegardens among three Amazonian villages. Economic Botany 53(3):312-326. dx.doi.org/10.1007/BF02866644

Marín-Corba, C., D. Cárdenas-López \& S. Suárez-Suárez. 2005. Utilidad del valor de uso en etnobotánica: Estudio en el departamento de Putumayo (Colombia). Caldasia 27(1):89-101. www.revistas.unal.edu.co/index.php/cal/ article/view/39315/41198

Morcote-Ríos, G. \& T. León Sicard. 2012. Estudio de semillas y carbón vegetal. Pp. 68-73 en Las Tierras Pretas del Igarapé Takana: Un sistema de cultivo precolombino en Leticia-Amazonas, Colombia. Editado por G. Morcote-Ríos \& T. León Sicard. Universidad Nacional de Colombia, Facultad de Ciencias, Bogotá, Colombia.

Nieto, J. 2006. Mujeres de la Abundancia. Tesis Maestría en Estudios Amazónicos. Universidad Nacional de Colombia, Sede Amazonia, Leticia, Amazonas, Colombia.

Padoch, C. \& W. De Jong. 1991. The house gardens of Santa Rosa: Diversity and variability in an Amazonian agricultural system. Economic Botany 45(2):166-175. dx.doi.org/10.1007/BF02862045

Paz Y Mino C., G., H. Blaslev \& R. Valencia. 1995. Useful lianas of the Siona-Secoya Indians from Amazonian Ecuador. Economic Botany 49(3):269-275. dx.doi. org/10.1007/BF02862346

Peña-Venegas, C., A. Mazorra, L. Acosta \& M. Pérez. 2009. Seguridad Alimentaria en Comunidades Indígenas del Amazonas: Ayer y hoy, Bogotá. Instituto Amazónico de Investigaciones Científicas (SINCHI), Leticia, Colombia.

Phillips, O. 1996. Some quantitative methods for analyzing ethnobotanical knowledge. Pp. 171-190 en Selected Guidelines for Ethnobotanical Research: A field manual. Editado por M. Alexiades. New York Botanical Garden, Bronx, New York, U.S.A. 
Phillips, O. \& A.H. Gentry. 1993. The useful plants of Tambopata, Peru: I. Statistical hypotheses tests with a new quantitative technique. Economic Botany 47(1):15-32. dx.doi.org/10.1007/BF02862203

Quinlan, M.B., R.J. Quinlan \& J.M. Nolan. 2002. Ethnophysiology and herbal treatments of intestinal worms in Dominica, West Indies. Journal of Ethnopharmacology 80(1):75-83. dx.doi.org/10.1016/S0378-8741(02)00002$\underline{8}$

R Software. 2011. Tinn-R Editor - GUI for $R$ Language and Environment. Versión 2.3.7.1. http://nbcgib.uesc.br/ lec/software/editores/tinn-r/en

Ribeiro, J., M. Hopkins, A. Vicentini, C. Sothers, M.J.M. Costa, M.A. Brito, I.H. Souza, I.G. Martinis, P.A. Lohmann, C. Assunção, E. Pereira, F. Silva, M. Mesquita \& L. Procópio. 1999. Flora da Reserva Ducke: Guia de identificação das plantas vasculares de uma floresta de terra firme na Amazônia Central. Royal Botanic Gardens, Kew, U.K.

Rudas-L., A. \& A. Prieto-C. 2005. Flórula del Parque Nacional Natural Amacayacu, Amazonas, Colombia. Monographs in Systematic Botany, Missouri Botanical Garden 99:1-680.

Sánchez, M., P. Duque, E. Miraña \& J. Miraña. 2001. Valoración del uso no comercial del bosque. Métodos en etnobotánica cuantitativa. Pp 179-224 en Evaluación de Recursos Vegetales no Maderables en la Amazonía Noroccidental. Editado por J.F. Duivenvoorden, $\mathrm{H}$. Balslev, J. Cavelier, C. Grandez, H. Tuomisto y R. Valencia. IBED, Universiteit van Ámsterdam, Amsterdam, The Netherlands.

Sierra, S. 2007. Caracterización Florística y Usos de las Especies de la Chagra en la Comunidad Indígena Mo- nilla Amena Kilometro 9.8 Leticia, Amazonas. Tesis en Ecología (Digital), Pontificia Universidad Javeriana, Bogotá, Colombia.

Sturges, H.A. 1926. The choice of a class-interval. Journal of the American Statistical Association 21:65-66. dx.doi. org/10.1080/01621459.1926.10502161

The Plant List. 2013. Version 1.1. www.theplantlist.org/. Consultado 20 enero 2014.

Tríana-Moreno, L.A., N.C. Rodríguez \& J. García. 2006. Dinámica del sistema agroforestal de chagras como eje de la producción indígena en el Trapecio Amazónico (Colombia). Agronomía Colombiana 24(1):158-169.

Tropicos.org. 2014. Tropicos, Botanical Information System at the Missouri Botanical Garden. www.tropicos.org Consultado 22 enero 2014.

van der Hammen, M. 1992. El Manejo del Mundo: Naturaleza y sociedad entre los Yukuna de la Amazonia Colombiana. Tropenbos International, Bogotá, Colombia.

Vásquez-M., R. 1997. Flórula de las Reservas Biológicas de Iquitos, Perú. Monographs in Systematic Botany, Missouri Botanical Garden 63:1-1046.

Vélez, G. \& J. Vélez. 1999. Sistema Agroforestal de las Chagras Indígenas del Medio Caquetá. Estudios en la Amazonía Colombiana, Volumen XVII. Primera edición. Tropenbos International, Bogotá, Colombia.

Wezel, A. \& J. Ohl. 2005. Does remoteness from urban centers influence plant diversity in homegardens and swidden fields?: A case study from the Matsiguenka in the Amazon rainforest of Peru. Agroforestry Systems 65(3):241-251. dx. doi.org/10.1007/s10457-005-3649-9 
Sierra \& Raz - Uso y Manejo de las Especies Sembradas en las Chagras de Dos Comunidades Murui-Muinane de la Amazonia Colombiana

\begin{tabular}{|c|c|c|c|c|c|c|c|c|c|c|c|c|c|c|c|c|c|c|c|c|c|c|c|c|c|c|c|c|c|}
\hline LT11 & & & & & & & & & & $F$ & & & & & & & & & & & & & & & & & & & \\
\hline LT10 & & & & & & & & & & & & & & & & & & & & 0 & & & & $\nabla$ & & & & & \\
\hline LT9 & & & & & & & & & - & $\forall$ & & & & & & & & & & & & & & & & & & م & \\
\hline LT8 & & & & & & & & & & $\cong$ & & & & & & & & & & & & & & & & & ما & & \\
\hline LT7 & & & & & & & & 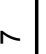 & $\theta$ & の & & & & & & & & & & & & & & & & & & & \\
\hline LT6 & & & & & & & & & & $\infty$ & $m$ & & & & & & & & $m$ & ما & $\wedge$ & $\theta$ & & & & & & & \\
\hline LT5 & & & & & & & & & & $\mp$ & & & & & & & & N & & & & & & 0 & & & & & \\
\hline LT4 & & $\sim$ & & & & & & & ه & $\sim$ & N & & & & & & - & & & & & & & & & & & & \\
\hline LT3 & & & & & & & & & & 0 & & & & & & & & & & & & & & & & & & & - \\
\hline LT2 & & & & & & & & $m$ & & $\infty$ & & & & & & & & & & & & & $\infty$ & & & & N & & \\
\hline LT1 & & & & & & & & & - & $\cong$ & & $\theta$ & & & or & 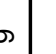 & & & & ما & & $m$ & & is & & & $\nabla$ & 0 & \\
\hline SR14 & & & & & & & & & N & N & & & & & & & & & & & & & & & & & & & \\
\hline SR13 & & & & & & & & & & $\infty$ & $m$ & & & 0 & & & & & & & ما & & & & & & م & & \\
\hline SR12 & & & & & & & & & & a & & & & & & & & & & & & & & & & & & & \\
\hline SR11 & & & & & & & & & $N$ & $\Lambda$ & & & & & & & & & & & & & & & & م & & & \\
\hline SR10 & م & & & & & & & & $m$ & $\stackrel{m}{=}$ & & & & & & & & & & & & & & 이 & $\theta$ & & & & \\
\hline SR9 & & & & & & s. & & & & ما & & & N & & & & & & & $N$ & & & & & & & & & \\
\hline SR8 & & & $m$ & & $m$ & & & & & $\mp$ & & & & & & & & & & & & $\Lambda$ & & & & & & & \\
\hline SR7 & & & & & & & & & & 0 & & $N$ & & & & & & $\sim$ & $\nabla$ & & & & & & & & & & \\
\hline SR6 & & & & & & & & & & & & & & & & & & & & & & & & & & & & & \\
\hline SR5 & & & & & & & & & & $\Lambda$ & & & & & $m$ & $n$ & $\sim$ & & & & & 으 & & & & & & & \\
\hline SR4 & & $m$ & & & & & & & & $\infty$ & $\theta$ & & & & & & & & & & & & & $\stackrel{m}{\square}$ & & & & & \\
\hline SR3 & & & & & & & & & & & & & & & & & & & & & & & & & & & & & \\
\hline SR2 & & & & $\nabla$ & & & & & & $\cong$ & & & & & & & & & & & & & & & & & & & \\
\hline SR1 & & & & & & & & & & & & & & & & & & & & & & & & & & & & & \\
\hline Origen & $z$ & $z$ & $z$ & $z$ & $z$ & $z$ & & $z$ & $z$ & $z$ & z & $z$ & $z$ & $z$ & $z$ & $z \mid z$ & $z$ & $z$ & $z$ & $z$ & $z$ & $z$ & -1 & $z$ & $z$ & $z$ & - & - & $z$ \\
\hline 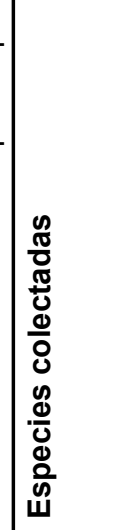 & 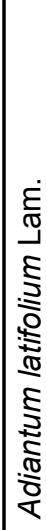 & 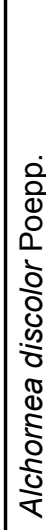 & 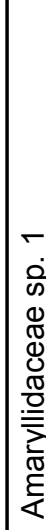 & 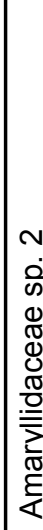 & 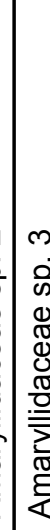 & 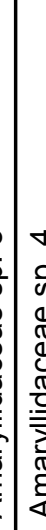 & 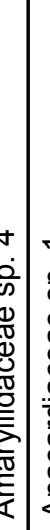 & 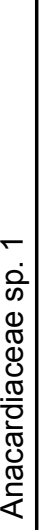 & 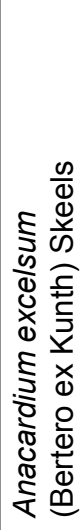 & 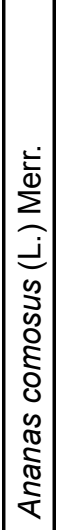 & - & 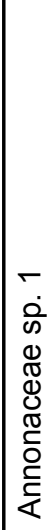 & 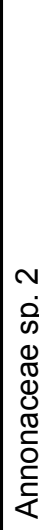 & 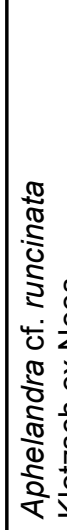 & 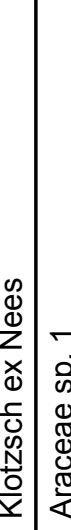 & 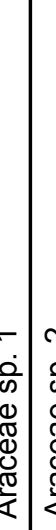 & 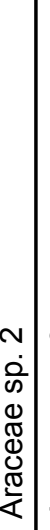 & $\begin{array}{l}m \\
\dot{0} \\
\infty \\
\Phi \\
\mathbb{D} \\
\mathbb{d} \\
0 \\
\frac{\pi}{2} \\
\frac{\pi}{<}\end{array}$ & $\begin{array}{l}+ \\
\dot{0} \\
\dot{0} \\
\mathbb{8} \\
\mathbb{8} \\
0 \\
\frac{\pi}{2}\end{array}$ & $\begin{array}{l}0 \\
\dot{0} \\
\infty \\
\mathbb{8} \\
\mathbb{8} \\
0 \\
\frac{\pi}{2}\end{array}$ & 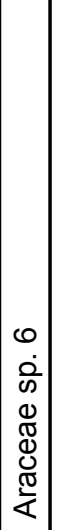 & 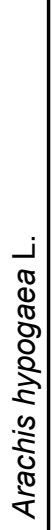 & 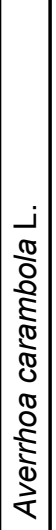 & 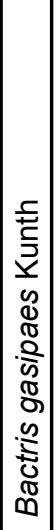 & 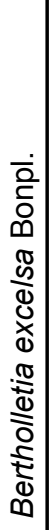 & 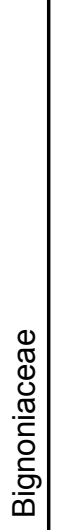 & 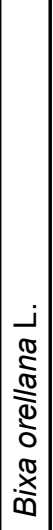 & 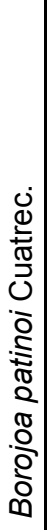 & 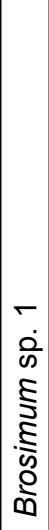 \\
\hline
\end{tabular}




\begin{tabular}{|c|c|c|c|c|c|c|c|c|c|c|c|c|c|c|c|c|c|c|c|c|c|c|c|c|c|c|c|}
\hline LT11 & & & & & & & & & & & $\nabla$ & & & & L & & & & & & & & & & & & \\
\hline LT10 & & & & & $\infty$ & & & & & & & & & & & & & & & & & $m$ & & & & & \\
\hline LT9 & & 0 & & & & & & & & & & c & $\infty$ & & & & & & & & & & & & & & \\
\hline LT8 & & & & & & & & & & & & & & & & & & & & & & & & & & & \\
\hline LT7 & & $\Lambda$ & & & & & & & & & & & & & & & & & & & & & & & & & \\
\hline LT6 & & $\infty$ & & & & & & & & & & & & & & & & & & & & & & & & & \\
\hline LT5 & & $m$ & & & & & & & & & & & & & & & & & & & & & & & & & \\
\hline LT4 & & & & & & & & & & & & & & & & & & & & 0 & & & & & & & \\
\hline LT3 & & $\nabla$ & & & & & & & & & & & & & & & & & & & & & & & & & \\
\hline LT2 & & $m$ & & $\Lambda$ & & & & & & & & & & & & & & & $\infty$ & & & & & & & & \\
\hline LT1 & & $m$ & & N & & & & & & & $m$ & & & & & ما & 0 & N & & & & & & 0 & & - & \\
\hline SR14 & & & & & & & & & & & & & & & & & & & & & & & & & & & \\
\hline SR13 & & 0 & & & & & $\forall$ & & & & & & & & & & & & & & & & & & & & \\
\hline SR12 & & & & & & + & & 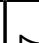 & & & & & & & & & & & & & & & & & & & \\
\hline SR11 & & & & & & & & & & & & & & & $\infty$ & & & & & & & & & & & & \\
\hline SR10 & & مـ & & & & & & $m$ & N & $c$ & & & & & & & & & & & 0 & & $\nabla$ & $N$ & $m$ & の & \\
\hline SR9 & & & & & & 0 & $m$ & $\nabla$ & & ר & & & & & & 0 & & & & & & & & & & & $\infty$ \\
\hline SR8 & & & & & & & & & & & & & & & & & & & & & & & & & & & \\
\hline SR7 & & & & & & & & & & & & & & & & & & $m$ & & & & & & & & & \\
\hline SR6 & $m$ & & In & & & & & & & & & & & & & & & & & & & & & & & & \\
\hline SR5 & & & & & & 0 & $\Lambda$ & $\nabla$ & $\Lambda$ & & & & & & & & & & & & & & & & & & \\
\hline SR4 & & N & & & & & & & & & & & & & & & & & & & & & & & & & \\
\hline SR3 & & & & & & & & & & $\infty$ & $\sim$ & & & & & & & & & $N$ & & $\nabla$ & & & & & \\
\hline SR2 & & & & & & & & & & & & & & & & & & & & & & & & & & & \\
\hline SR1 & & & & & & & & & & & & & & - & & & & & & & & & & & & & \\
\hline Origen & $z$ & $z$ & $z$ & $z$ & $z$ & $z$ & $z$ & $z$ & $z$ & - & - & -12 & $z$ & - & - & - & - & $z$ & - & $z$ & $z$ & $z$ & $z$ & $z$ & - & $z$ & - \\
\hline 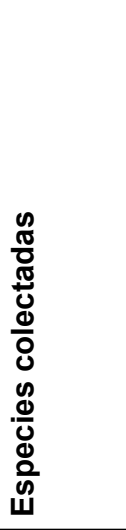 & 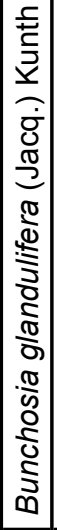 & 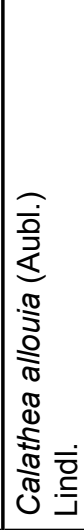 & 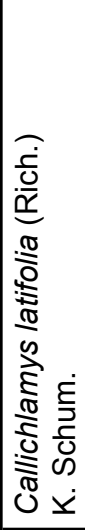 & 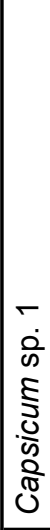 & 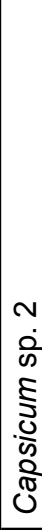 & $\begin{array}{l}m \\
\text { के } \\
\text { के } \\
\frac{5}{3} \\
\frac{0}{w} \\
\frac{2}{\delta} \\
0\end{array}$ & 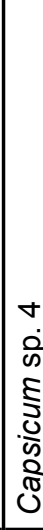 & $\begin{array}{l}10 \\
0 \\
0 \\
5 \\
5 \\
\frac{0}{5} \\
0 \\
0 \\
0\end{array}$ & {$\left[\begin{array}{l}0 \\
0 \\
0 \\
5 \\
\frac{0}{0} \\
\delta \\
0\end{array}\right.$} & 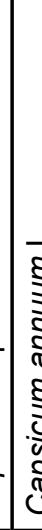 & 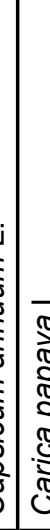 & $\begin{array}{l}j \\
0 \\
0 \\
0 \\
0 \\
0 \\
0 \\
0 \\
0 \\
0 \\
\vdots\end{array}$ & 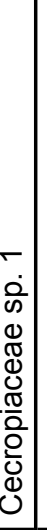 & 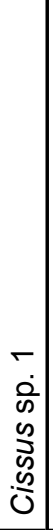 & 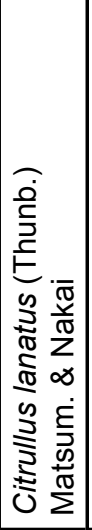 & 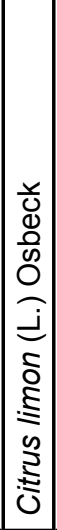 & 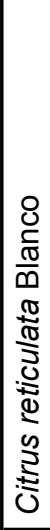 & 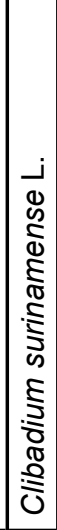 & 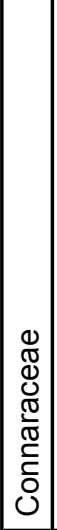 & 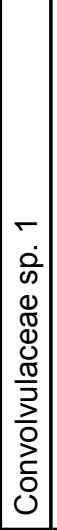 & 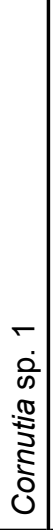 & 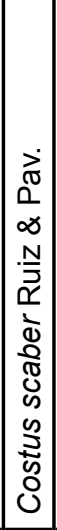 & 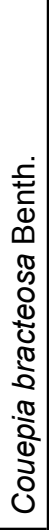 & 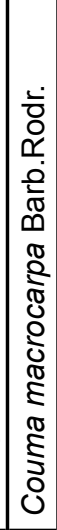 & $\begin{array}{l} \\
\\
- \\
0 \\
0 \\
0 \\
0 \\
0 \\
0 \\
0 \\
0 \\
\frac{\pi}{2} \\
\\
0 \\
0 \\
0 \\
0 \\
0\end{array}$ & 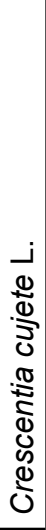 & 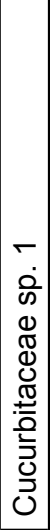 \\
\hline
\end{tabular}


Sierra \& Raz - Uso y Manejo de las Especies Sembradas en las Chagras de Dos Comunidades Murui-Muinane de la Amazonia Colombiana

\begin{tabular}{|c|c|c|c|c|c|c|c|c|c|c|c|c|c|c|c|c|c|c|c|c|c|c|c|c|c|c|c|}
\hline LT11 & & & & & & & $\mp$ & & & & & & & & & & & & & & & & & & & & \\
\hline LT10 & & & & & & & & & & & & & & & & & & & & & & & & & & & \\
\hline LT9 & & & & & & & $F$ & & & N & & & & & & & & & & & ص & & & & & & \\
\hline LT8 & & $\nabla$ & & & 0 & & & & & & & & & & & & & & & & & & & & & & \\
\hline LT7 & & & & & $\theta$ & & & & & & & & - & & & & & & & & & & & & & & \\
\hline LT6 & & & & & ما & & 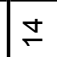 & & & & & & & & & & & & & & & & & & & & \\
\hline LT5 & & & & & ( & & $\stackrel{\oplus}{\square}$ & & & $m$ & & & & & & & & & & & & & & & & & \\
\hline LT4 & & & & & $\Lambda$ & & & & & & & & & & & & & & & & & & & & & & \\
\hline LT3 & & & & & & & & & & & & & & & & & & & & & & & & & & & \\
\hline LT2 & & $\infty$ & & & $F$ & & $\stackrel{m}{=}$ & & $\infty$ & & & & & $m$ & & & & & & & & & & & & & \\
\hline LT1 & & 0 & & & $\Lambda$ & & 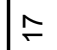 & & $\sigma$ & & & & - & & $\theta$ & & & & & & & & & & & & \\
\hline SR14 & & & & & & & & & & & & & & & & & & & & & & & & & & & \\
\hline SR13 & & & & & 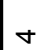 & & & $\infty$ & & & & & & & & & & & & & & & & & & & \\
\hline SR12 & & & & & & & $\stackrel{\simeq}{\simeq}$ & $\stackrel{\circ}{\circ}$ & & & & $N$ & & & & & & & & & & & & & & & \\
\hline SR11 & م & & & & & & & & & & & & & & & & & & & & & & & & & & \\
\hline SR10 & & & & & & & & a & & & & & & & $\Lambda$ & & & & & & & & & & & & \\
\hline SR9 & & & & & $\stackrel{\simeq}{\simeq}$ & & & & & & & & & & & & & & & & & & & & & & \\
\hline SR8 & & $\Lambda$ & & & & 0 & & & & & & & & & & & $\infty$ & & & 0 & & & & & & & م \\
\hline SR7 & & & & ما & 0 & & & $\stackrel{0}{\circ}$ & & & & & & & & & & & & & & & & & & $\nabla$ & \\
\hline SR6 & & & $m$ & & & & & 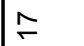 & & & & & & & & & & & & & & & & & 0 & & \\
\hline SR5 & & & & & & & 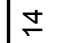 & $\bar{N}$ & & & & & & & & & & & & & & & & & & & \\
\hline SR4 & $m$ & & & & م & & & & & & o & & & & & - & & $N$ & & & & & & เ & & & \\
\hline SR3 & & & & & & & & & & & & & & & & & & & & & & $\Lambda$ & $m$ & & & & \\
\hline SR2 & & & & & & & & & & & & & & & & & & & & & & & & & & & \\
\hline SR1 & & & & & & - & & & & & & & & & & & & & - & & & & & & & & \\
\hline Origen & $z$ & - & z & $z$ & $z$ & $z$ & $z$ & $z$ & $z$ & $z$ & $z$ & $z$ & z & - & - & $z$ & $z$ & $z$ & - & $z$ & $z$ & z & $\mathrm{z}$ & 之 & 之 & z & 号 \\
\hline 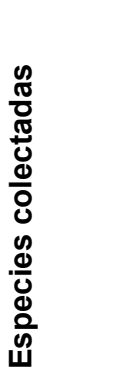 & 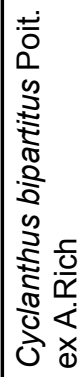 & 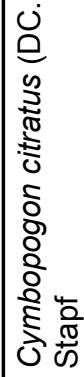 & 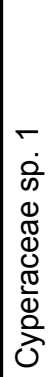 & $\begin{array}{l} \\
\text { in } \\
\mathbb{8} \\
\mathbb{1} \\
0 \\
0 \\
.0 \\
0\end{array}$ & 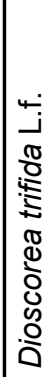 & 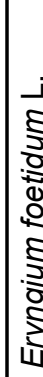 & 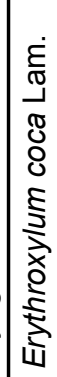 & 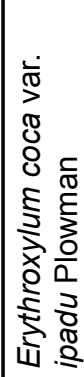 & 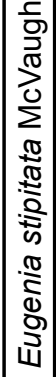 & 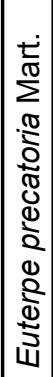 & 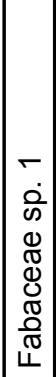 & 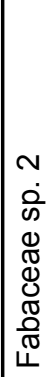 & $\begin{array}{l}- \\
- \\
0 \\
\text { की } \\
\text { S } \\
\text { O0 } \\
\text { iv }\end{array}$ & 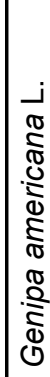 & 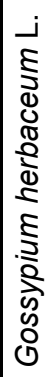 & 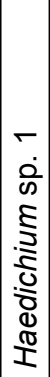 & 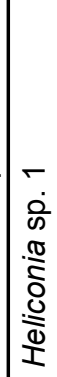 & 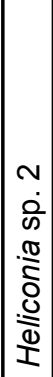 & 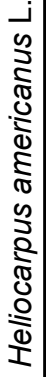 & \begin{tabular}{|c} 
\\
- \\
- \\
0 \\
0 \\
0 \\
$\frac{0}{2}$ \\
$\frac{\pi}{2}$ \\
$\frac{0}{0}$ \\
$\frac{0}{1}$
\end{tabular} & 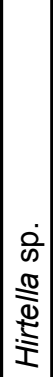 & 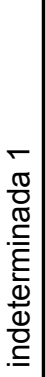 & 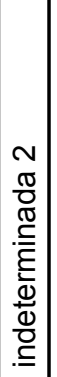 & 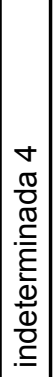 & 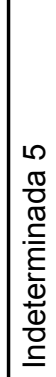 & 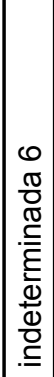 & 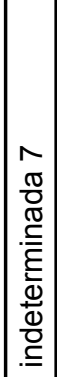 \\
\hline
\end{tabular}




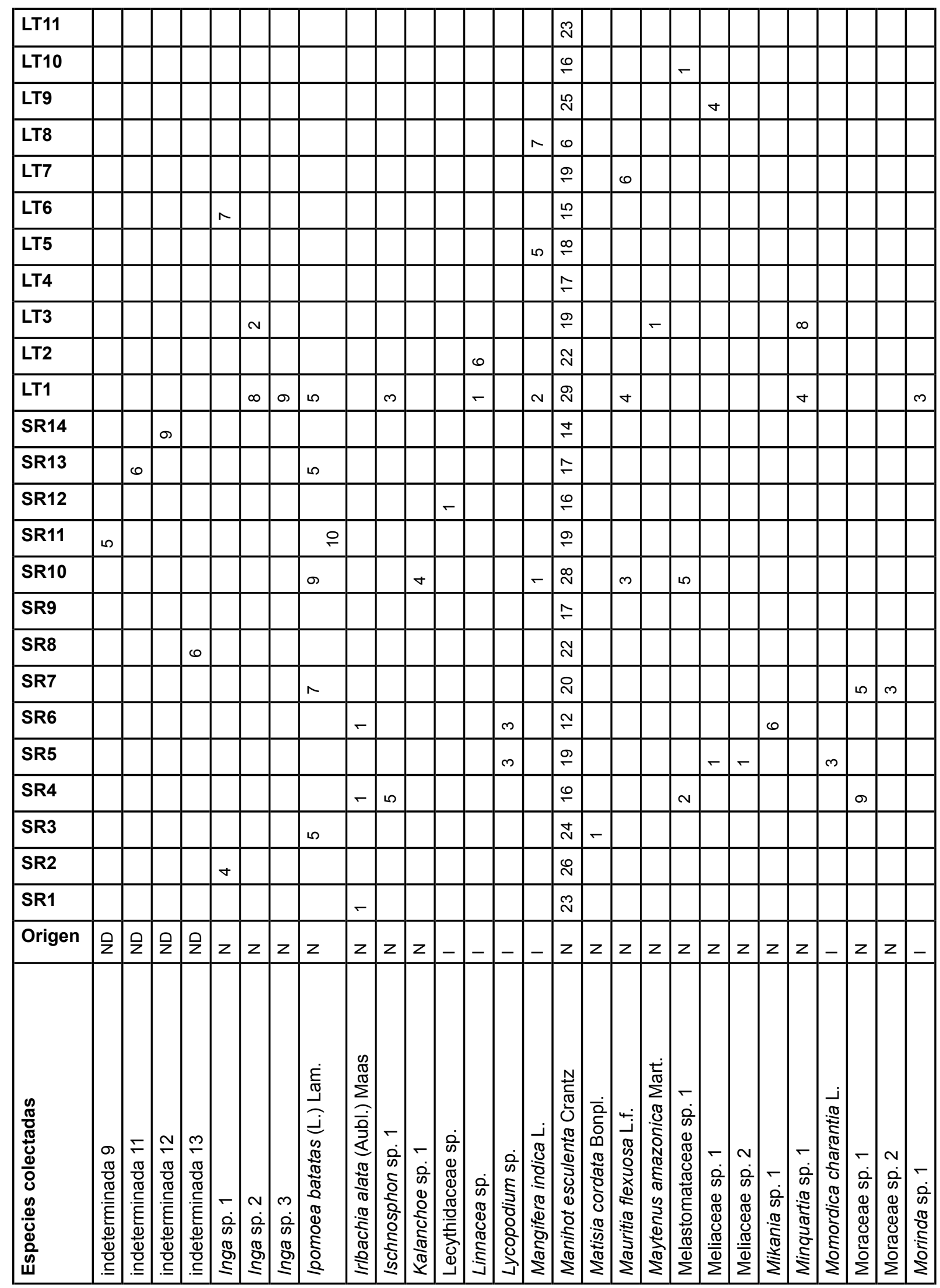


Sierra \& Raz - Uso y Manejo de las Especies Sembradas en las Chagras de Dos Comunidades Murui-Muinane de la Amazonia Colombiana

\begin{tabular}{|c|c|c|c|c|c|c|c|c|c|c|c|c|c|c|c|c|c|c|c|c|c|c|c|c|c|c|c|}
\hline LT11 & $F$ & & & $\stackrel{20}{2}$ & & & & & & & & & & & & & & & מ & & & & & & & & \\
\hline LT10 & & & & & & $m$ & & & & & & & & & & & & $\nabla$ & & & & & & & & & \\
\hline LT9 & & & & & & & 0 & & & & $\theta$ & & & & & & & & & $m$ & & & & & & & \\
\hline LT8 & & & & & & & & & & & & & & & & & & & & & & & & & & & - \\
\hline LT7 & & & & & & & & & & $\wedge$ & $m$ & & & & & & & & & 0 & & $\Lambda$ & & $\infty$ & & & \\
\hline LT6 & & & & & & & & & & 0 & & & & & & & & & & $\infty$ & & & & & & & \\
\hline LT5 & & & & & & & $m$ & & & & & & & & & & & & & $\theta$ & & $\infty$ & & $\nabla$ & & & \\
\hline LT4 & $\cong$ & & & & & & & & の & & & & $m$ & & & & & & & & & & & & & & \\
\hline LT3 & & & & & & & & & & & & & & & & & & & & & & م & & & & & \\
\hline LT2 & 0 & & & & & & & & & & & & & & & & & & & & & & & & & & $r$ \\
\hline LT1 & & & & & & & & 0 & & 10 & $\infty$ & & $N$ & & & & & & & م & $\sigma$ & & & $\sim$ & & & \\
\hline SR14 & $\Lambda$ & & & & & & & & & & & & & & & & & & & & & & & & & & \\
\hline SR13 & 0 & & & & 0 & & & & & & $N$ & & & & & & & & & $m$ & & & & & & & \\
\hline SR12 & 0 & & & & & & & & $r$ & & & & $\sigma$ & & & & & & & & & & & & & & \\
\hline SR11 & $m$ & & & & & & & & & $\theta$ & & م & 0 & & & & & & & N & & & & & & & \\
\hline SR10 & N & & & & & & & & & & & & & & & & & & & $m$ & & & & & & & \\
\hline SR9 & 움 & & & & $\theta$ & & & & & & - & & & & & & $\Lambda$ & & & & & & o & & & & \\
\hline SR8 & & & & & & & & & & & & & & & & & & & & & & & & & & & \\
\hline SR7 & & & & & & & & & & & & & & & & & & & & $\infty$ & & N & & & & N & \\
\hline SR6 & $\sigma$ & $m$ & (1) & & & & & & & & & & & & & $\infty$ & 0 & & & & & & & & & & \\
\hline SR5 & & & & $\Lambda$ & & & & & & & & & & & & & & 0 & & & & & & & & & \\
\hline SR4 & & & & & & & & N & & & & & & & & & & & & $\theta$ & & & & & & & \\
\hline SR3 & 0 & & & & & & & & & $m$ & & & & $m$ & $N$ & & $\nabla$ & & & & & & & & $\sim$ & & \\
\hline SR2 & & & & & & & & & & & & & & & & & & & & & & & & & & & \\
\hline SR1 & & & & & & & & & & & & & & & & & & & & & & & & & & & \\
\hline Origen & - & $z$ & $z$ & $z$ & - & z & z & $z$ & - & $z$ & - & - & - & $z$ & $z$ & $z$ & - & $z$ & 号 & $z$ & z & $z$ & $z$ & $z$ & $z$ & $z$ & $z$ \\
\hline 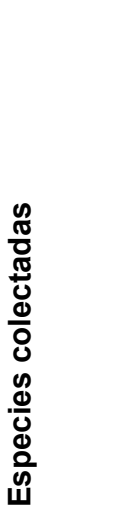 & 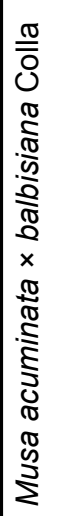 & 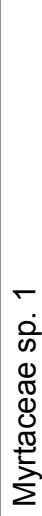 & $\begin{array}{l}- \\
\dot{0} \\
\dot{0} \\
\mathbb{8} \\
\mathbb{8} \\
\dot{0}\end{array}$ & 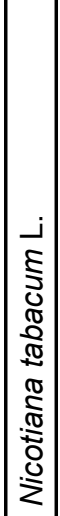 & 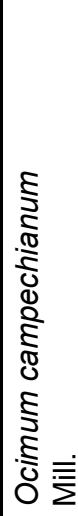 & 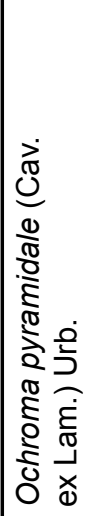 & 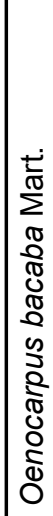 & 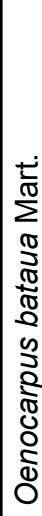 & 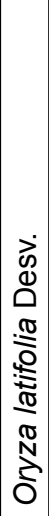 & 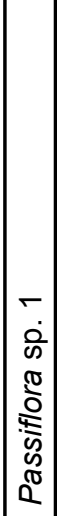 & 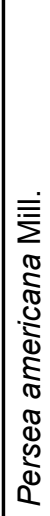 & 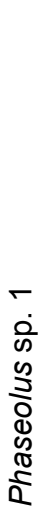 & 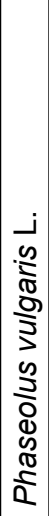 & 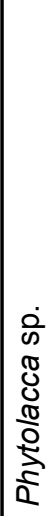 & 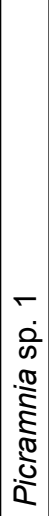 & $\begin{array}{l}N \\
\text { on } \\
\frac{1}{\Phi} \\
\frac{2}{2} \\
2\end{array}$ & 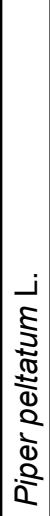 & 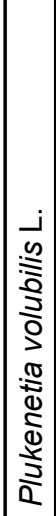 & \begin{tabular}{|l} 
\\
\\
- \\
0 \\
0 \\
0 \\
0 \\
0 \\
$\mathbb{0}$ \\
$\mathbb{0}$ \\
0 \\
0 \\
0 \\
0 \\
0
\end{tabular} & 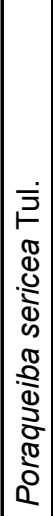 & 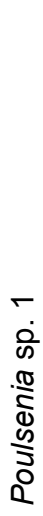 & $\begin{array}{l}- \\
\vdots \\
0 \\
0 \\
0 \\
\delta \\
0 \\
\vdots \\
0 \\
0\end{array}$ & 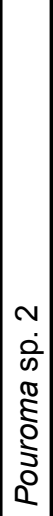 & 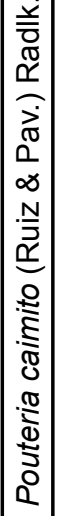 & 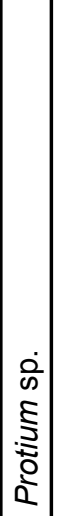 & 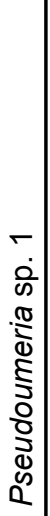 & $\begin{array}{l}- \\
\frac{0}{\omega} \\
\frac{5}{5} \\
\frac{5}{2} \\
\frac{5}{2}\end{array}$ \\
\hline
\end{tabular}




\begin{tabular}{|c|c|c|c|c|c|c|c|c|c|c|c|c|c|c|c|c|c|c|c|c|c|c|c|}
\hline LT11 & & & & & & $\infty$ & & & & & & & & & & & & & & & & & \\
\hline LT10 & & & & & & & & - & & & & & & & & & & & & & & & \\
\hline LT9 & & م & & & 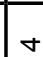 & & & & & & & & & 0 & - & & & & & & $m$ & & \\
\hline LT8 & & & & & & $\stackrel{m}{=}$ & & & & & & & & & & & & & $\infty$ & & & & \\
\hline LT7 & & 0 & & & & & & & & & $m$ & & & & & & & & 0 & & ما & & \\
\hline LT6 & & & & & & & & & & & $\theta$ & & & & & & & & $\theta$ & & & & \\
\hline LT5 & - & & & & & & & & & & & & & & & & & & ما & & & & \\
\hline LT4 & & & & & & & & & & & & & & & & & & & & & & & \\
\hline LT3 & & $\theta$ & & & & & & & & & & & & & & & & & & & & & \\
\hline LT2 & & & & $m$ & & ما & & & & & $\Lambda$ & & & & & & - & $m$ & & & N & & \\
\hline LT1 & & $m$ & $\infty$ & & & & & & & & o & & $m$ & & & & & & $\sim$ & ما & 0 & & \\
\hline SR14 & & & & & & & & & & & & & & & 0 & & & & & & & & \\
\hline SR13 & & & & & & & & & & & $\infty$ & - & & & & & & & & & & & \\
\hline SR12 & & & & & & & & & & & & & & & & & & & & & & & \\
\hline SR11 & & & & & & & & & & & $F$ & & & & & & & & & & & & L \\
\hline SR10 & & & & $\nabla$ & 0 & & $\nabla$ & & & & & & & & & & & & & & & & \\
\hline SR9 & & & & & & & & & & & & & & & & & & & & & & $\infty$ & \\
\hline SR8 & & & & & & & & & & & & & & & & & & & & & & & \\
\hline SR7 & & & & & ما & & & & & & & & & & & & & & & & & & \\
\hline SR6 & & & & & & & & & & & & & & & & & & & & & & & \\
\hline SR5 & & & & & & & & & & $\infty$ & 10 & ما & & & $N$ & & & & & & & & \\
\hline SR4 & & & & & & 0 & & & L & 0 & & $\theta$ & & & & 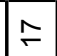 & & & & 0 & & & $\sim$ \\
\hline SR3 & & & & & & ما & & & & & $\Lambda$ & & & & & & & & & & & & \\
\hline SR2 & & & & & & & & & & & & & & & & & & & & $\Lambda$ & & & \\
\hline SR1 & & & & & & & & & & & o & & & & & & & & & & & & \\
\hline Origen & $z$ & $z$ & $z$ & $z$ & $z$ & z & - & 号 & - & - & - & - & $z$ & - & $z$ & - & $z$ & $z$ & $z$ & $z$ & $\bar{z}$ & - & - \\
\hline 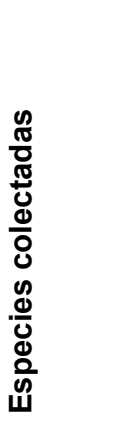 & 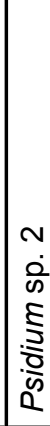 & 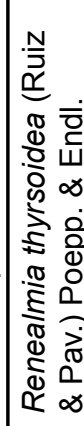 & 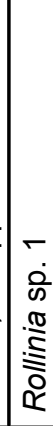 & 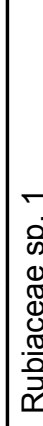 & 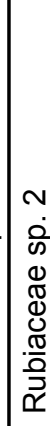 & 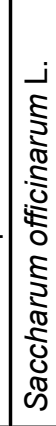 & 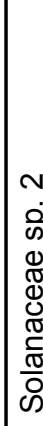 & 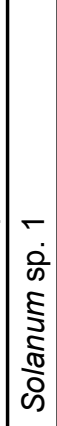 & 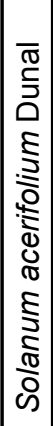 & 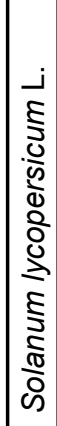 & 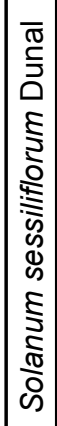 & 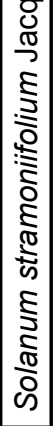 & 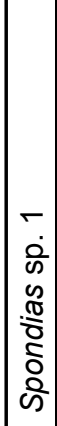 & 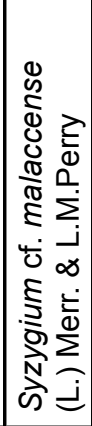 & 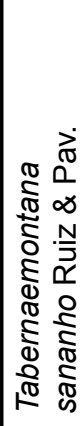 & 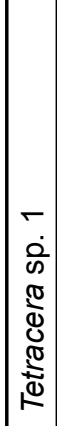 & 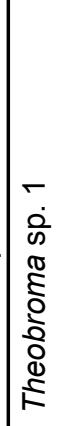 & 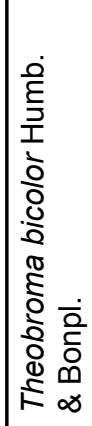 & 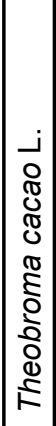 & 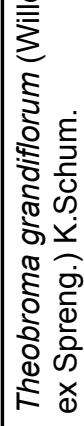 & 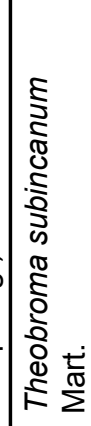 & 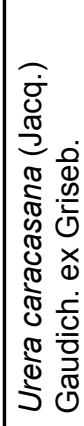 & 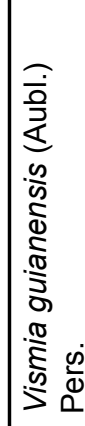 \\
\hline
\end{tabular}


Sierra \& Raz - Uso y Manejo de las Especies Sembradas en las Chagras de Dos Comunidades Murui-Muinane de la Amazonia Colombiana

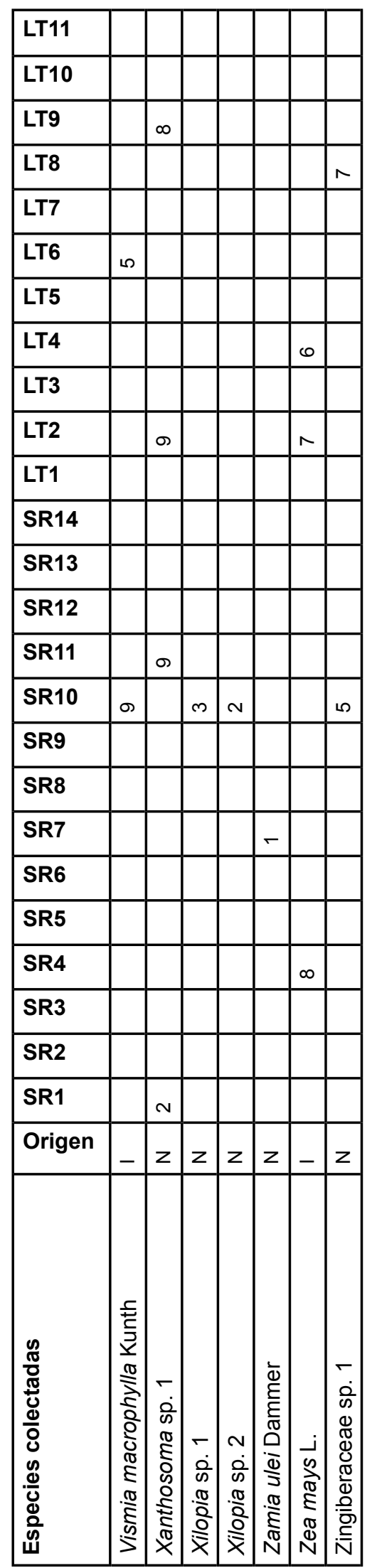


